\title{
VISCOUS FLOW WITH LARGE FREE SURFACE MOTION*
}

\author{
Antonio HUERTA** and Wing Kam LIU \\ Department of Mechanical Engineering, The Technological Institute, Northwestern University, \\ Evanston, IL 60208, U.S.A
}

Received 6 July 1987

Revised manuscript received 12 December 1987

\begin{abstract}
An arbitrary Lagrangian-Eulerian (ALE) Petrov-Galerkin finite clement technique is developed to study nonlinear viscous fluids under large free surface wave motion. A review of the kinematics and field equations from an arbitrary reference is presented and since the major challenge of the ALE description lies in the mesh rezoning algorithm, various methods, including a new mixed formulation, are developed to update the mesh and map the moving domain in a more rational manner. Moreover, the streamline-upwind/Petrov-Galerkin formulation is implemented to accurately describe highly convective free surface flows. The effectiveness of the algorithm is demonstrated on a tsunami problem, the dam-break problem where the Reynolds number is taken as high as 3000 , and a large-amplitude sloshing problem.
\end{abstract}

\section{Introduction}

Although the finite element method (FEM) is one of the most powerful and sophisticated numerical techniques available, most of its early developments were applied to structural analysis and it was not until the late 1960's that finite element techniques were applied to potential flow problems. Recently, considerable finite element research is being devoted to viscous flows, transport processes, fluid-structure interaction, compressible inviscid flows, and free surface flows, among others. However, the application of finite element analysis to free surface viscous flows, such as rock slides, snow avalanches, breaking of a dam or mine tailings impoundments, and polymer processing flows (e.g. extrusion, coating, injection molding, etc.), is still rudimentary. This paper is devoted to the development of arbitrary LagrangianEulerian (ALE) techniques for viscous flows with free surface.

The kinematic description (i.e. the relationship between the moving fluid and the finite element grid) is extremely important in multidimensional fluid dynamics problems. Two classical descriptions are used in continuum mechanics. The first is Lagrangian, in which the mesh points coincide with the material particles. In this description, no convective effects appear and this simplifies considerably the numerical calculations; moreover, a precise definition of moving boundaries and interfaces is obtained. However, the Lagrangian description does not handle satisfactorily the material distortions that lead to element entanglement.

${ }^{*}$ The support of A. Huerta by a Catalan Fellowship and the Royal E. Cabell from Northwestern University, and the support of Wing K. Liu by the National Science Foundation are gratefully acknowledged.

** Present address: E.T.S. de Ingenieros de Caminos, Universidad Politécnica de Cataluña, Barcelona, Spain. 
On the other hand, the second description is the Eulerian viewpoint, which allows strong distortions without problems because the mesh is fixed with respect to the laboratory frame and the fluid moves through it. However, this latter approach presents two important drawbacks: (i) convective effects, which introduce numerical difficulties, a: ise due to the relative movement between the grid and the particles; and (ii) sophisticated mathematical mappings between the stationary and moving boundaries are required.

Because of the shortcomings of purely Lagrangian and Eulerian descriptions, arbitrary Lagrangian-Eulerian (ALE) techniques were developed, first in finite differences by Noh [40] and Hirt et al. [16], among others, and then in finite elements by Donea et al. [11], Belytschko and Kennedy [2], Hughes et al. [22], and Donea [9]. This new approach is based on the arbitrary movement of the reference frame, which is continuously rezoned in order to allow a precise description of the moving interfaces and to maintain the element shape. Convective terms are still present in the ALE equations, but the ability to prescribe the mesh movement may allow them to be reduced.

However, it is sometimes very difficult to optimize the mesh mapping and at the same time decrease the convective velocities. In these cases, the grid rezoning has priority, and numerical techniques are implemented to overcome the spurious spatial oscillations created by the

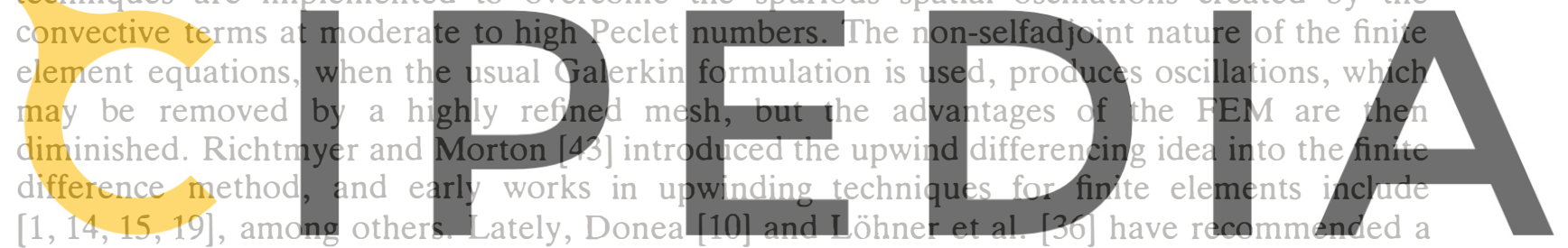
Taylor-Galerkin approach for the convection-dominated equations, whereas Brooks and

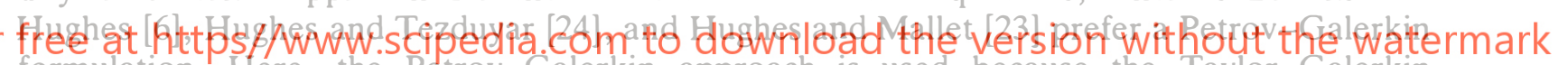
formulation. Here, the Petrov-Galerkin approach is used because the Taylor-Galerkin technique is difficult to apply to nonlinear convection-diffusion equations such as the

Navier-Stokes equations for non-Newtonian fluids. It is important to notice that the mesh updating equations presented herein are of the pure convective type. Hence, the mesh rezoning equations are also formulated using the streamline-upwind/Petrov-Galerkin technique.

The outline of the present paper is arranged as follows. First, the notation and kinematics of the ALE description are introduced in order to derive the governing equations. The field equations (continuity and equilibrium) are revised in referential form; then, mesh updating equations are formulated. Once the partial equations governing the fluid and mesh motions are known, their finite element formulation is developed. This leads to a system of differential equations which is solved by a predictor-multicorrector algorithm. Finally, three free surface flow problems are studied to demonstrate the effectiyeness of the present development.

\section{Kinematics in the ALE description}

\subsection{Review of the ALE description}

Two classic viewpoints are considered to describe the motion of a continuous medium. The first is Lagrangian, in which the material region and the coordinates of any point are denoted 
by $R_{X}$ and $X$, respectively. In the second, known as Eulerian, the spatial region is symbolized by $R_{x}$ and the spatial coordinates by $x$. In the ALE description, the computational frame is a reference independent of the particle movement and which may be moving with an arbitrary velocity in the laboratory system; the continuum view from this reference is denoted as $R_{\chi}$, and the coordinates of any point are denoted as $\boldsymbol{\chi}$. Figure 1 is a schematic diagram of the above-mentioned domains with the one-to-one mappings $\Phi$ and $\Psi$ relating $R_{x}$ to $R_{x}$ and $R_{X}$, respectively. In fact, $\boldsymbol{\Phi}$ and $\boldsymbol{\Psi}$ can be represented, symbolically, as

and

$$
\begin{aligned}
& R_{x} \times\left[0, \infty\left[\rightarrow R_{x},\right.\right. \\
& (\chi, t) \mapsto \Phi(\chi, t)=x,
\end{aligned}
$$

$$
\begin{aligned}
& R_{X} \times\left[0, \infty\left[\rightarrow R_{X},\right.\right. \\
& (X, t) \mapsto \Psi(X, t)=X,
\end{aligned}
$$

where $t$ denotes time. Note that a referential description corresponds to an Eulerian viewpoint when $\Phi$ is the identity function at any time. On the other hand, if $\Psi$ is the identity function, the reference domain, $R_{x}$, is equivalent to
description is used.
Both the materia and spatial dom din are
throughout this formulation, it is con enien,
referential form. Consider a physical property,
view of the mappin defined by (1) and (2),
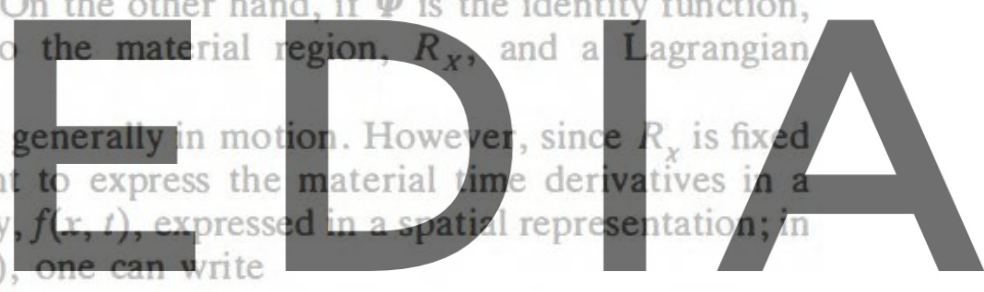

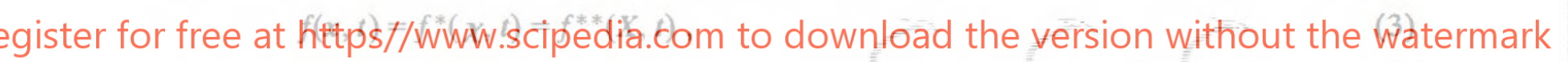

where * and ** denote "with respect to $X$ and $X^{\text {*2 }}$, respectively, and by composition of mappings $\left({ }^{\circ}\right)$,

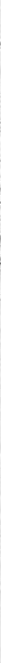

Fig. 1. Schematic diagram of domains and mappings for the arbitrary Lagrangian-Eulerian description. 


$$
f^{*} \quad f \circ \boldsymbol{\Phi} \text { and } f^{* *} f \circ \boldsymbol{\Phi} \circ \boldsymbol{\Psi}^{-1} .
$$

Differentiating this physical property with respect to $t$, holding $X$ fixed (i.e. a material derivative of $f^{* *}$ ), and using the chain rule, we get

where

$$
\left.\frac{\partial f^{* *}}{\partial t}(\boldsymbol{X}, t)\right|_{\boldsymbol{X}}=\left.\frac{\partial f^{*}}{\partial t}(\boldsymbol{X}, t)\right|_{\boldsymbol{X}}+w_{i} \partial f^{*}(\chi, t)
$$

$$
w_{i}=\left.\frac{\partial \chi_{i}}{\partial \imath}\right|_{x}
$$

and $w$ is defined as the particle velocity with respect to the referential coordinates. Note that standard indicial notation is adopted; lower-case subscripts denote the components of a tensor and repeated indices imply summations over the appropriate range (number of spatial dimensions in the particular case of the preceding equations). If the physical property is the spatial coordinate $x,(3)$ and (5) yield
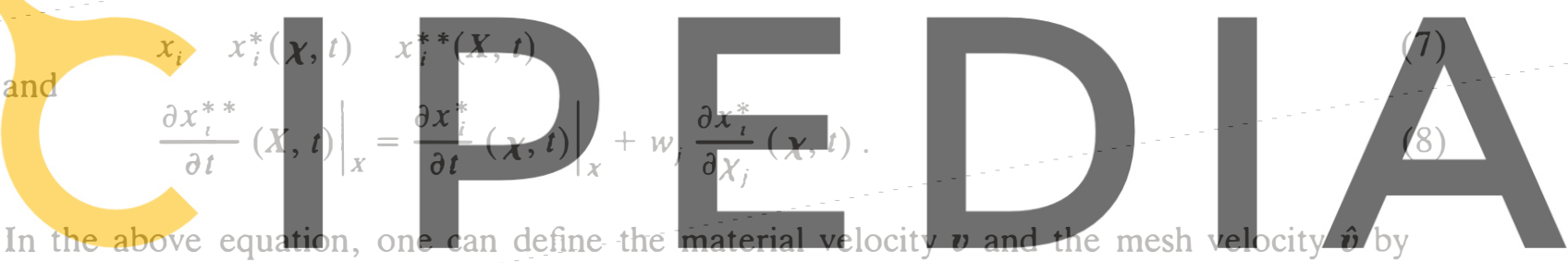

egister for free at htţिs and

$$
\hat{v}_{i}=\left.\frac{\partial x_{i}^{*}}{\partial t}(\chi, t)\right|_{\chi}
$$

respectively. Therefore, (8) may be rewritten as

or

$$
v_{i}=\hat{v}_{s}+w_{j} \frac{\partial x_{i}^{*}}{\partial x_{i}}
$$

where

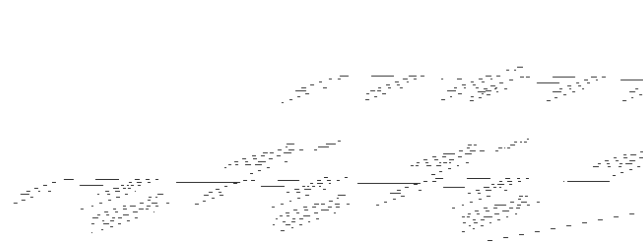

is the convective velocity. Equations $(10)-(12)$ were originally developed by Hughes et al. [22] and they are the basis for the automatic mesh rezoning that will be discussed later. Finally, substituting (12) into (5) and applying the chain rule yields the relationship between the material time derivative and the referential time derivative:

$$
\left.\frac{\partial f^{* *}}{\partial t}(X, t)\right|_{X}=\left.\frac{\partial f^{*}}{\partial \ell}(\chi, t)\right|_{\chi}+c_{i} \frac{\partial f}{\partial x_{i}}(x, t) .
$$




\subsection{Lagrangian versus referential updates}

Note that in the Lagrangian description, the updating of any physical property is simple and it is done following always the same particle. Using a Taylor-series expansion in time, first-order accuracy gives

$$
f^{* *}(X, t+\mathrm{d} t)=f^{* *}(X, t)+\left.\mathrm{d} t \frac{\partial f^{* *}}{\partial t}(\boldsymbol{X}, t)\right|_{X}+\cdots
$$

Similarly, in a referential description the previous equation is written as

$$
f^{*}(\chi, t+\mathrm{d} t)=f^{*}(\chi, t)+\left.\mathrm{d} t \frac{\partial f^{*}}{\partial t}(\chi, t)\right|_{\chi}+\cdots,
$$

or, by referring everything to the original particle, as
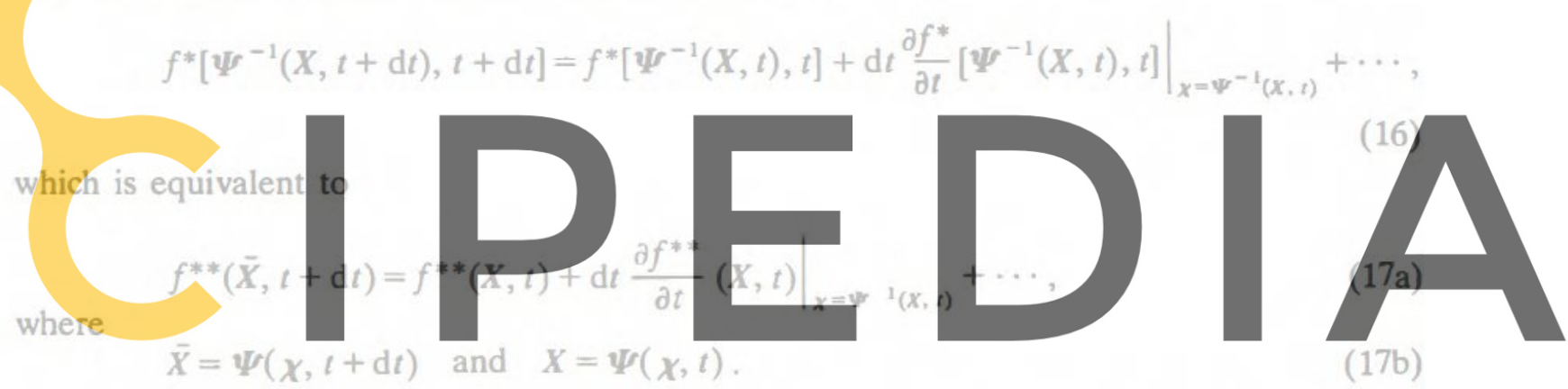

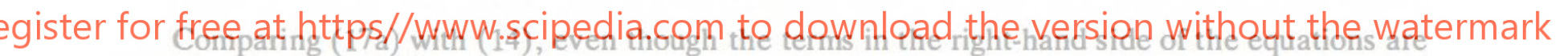

the same, $X$ and $\bar{X}$ are two different material particles which, at $t$ and $t+\mathrm{d} t$, respectively, have

the same referential coordinates. Therefore, in a referential description, a simple updating technique, such as (15), cannot be used for material point related variables, such as state variables in path-dependent materials; however, for homogeneous materials with no memory, such as generalized Newtonian fluids, (15) can be implemented with no further complications. Examples of ALE techniques applied to path-dependent materials may be found in [29, 32].

\subsection{Reynolds transport in referential form}

In order to establish the integral form of conservation laws in ALE, the material derivative of a scalar property, $G(t)$, is studied, Let $G$ be defined by the volume integral

$$
G(t) \int_{V_{x}}^{t} f^{*}(x, t) \mathrm{d} \Omega,
$$

with the arbitrary control volume $V_{x}$ being included in $R_{x}$. Making use of Gauss' theorem and classical algebra [9], the material time derivative of (18) bećomes

$$
\overline{\partial t} \quad \mathrm{~d} \Omega+\mathrm{c}
$$


where $\boldsymbol{w}$ is the particle velocity viewed from the fixed reference, defined in (6), and $\hat{\boldsymbol{n}}$ is the outer normal to the surface of $V_{\chi}$ (i.e. $\partial V_{\chi}$ ). Physically, (19) states that the rate of change of $G(t)$ is equal to the sum of the amount instantaneously created in $V_{x}$ and the flux through the boundary surface $\partial V_{x}$ induced by the relative movement of the reference frame. The particularizations of (19) to the Lagrangian and Eulerian method are straightforward; set $\boldsymbol{\chi}=\boldsymbol{X}$ and $\boldsymbol{w}=\mathbf{0}$ for the Lagrangian approach, and $\boldsymbol{\chi}=\boldsymbol{x}$ with $\boldsymbol{w}=\boldsymbol{v}$ for the Eulerian approach.

\section{Initial/boundary value problem}

\subsection{Field equations in the ALE method}

To simplify the subsequent developments, the 'star' notation used to differentiate the three domains (i.e. $R_{x}, R_{X}$, and $R_{x}$ ) will be dropped. Appendix A presents the derivation of the continuity and equilibrium equations in both Lagrangian and Eulerian descriptions, making
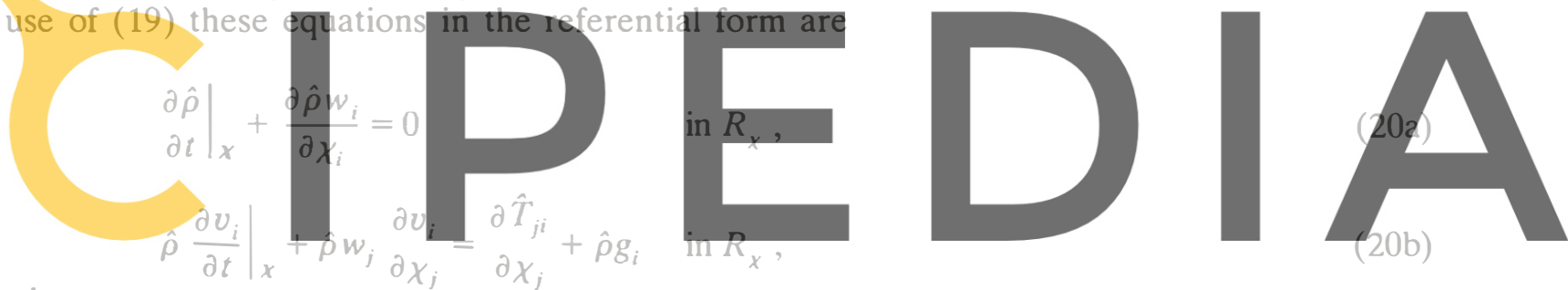

egister for free at https///WwiW.scipedia.com to download the version without the watermark

$$
\begin{aligned}
& \hat{\rho}(\chi, t)=\hat{J} \rho(x, t), \\
& \hat{T}_{i j}=\hat{J} \frac{\partial \chi_{i}}{\partial x_{k}} \sigma_{k j},
\end{aligned}
$$

$\rho$ is the fluid density, $\boldsymbol{g}$ is the acceleration of gravity, and $\boldsymbol{\sigma}$ and $\hat{\boldsymbol{T}}$ are the Cauchy and first Piola-Kirchhoff stress tensors, respectively.

Equations (20) are seldom applied in finite element methods because the stress term in (20b) presents similar difficulties as in the total Lagrangian formulation (i.e. a nonsymmetric stress tensor or the computational burden created by the use of the second Piola-Kirchhoff stress tensor). Moreover, the constitutive relationship is usually written in terms of the Cauchy stress. Therefore, another formulation is suggested [22].

The simplest approach for treating the stress term is to employ the Cauchy stress formulation. The weak form of the field equations is obtained by integration over the spatial region, $R_{x}$, instead of the referential one, $R_{x}$. However, all of the functions are written in terms of $\boldsymbol{\chi}$, not $\boldsymbol{x}$, because the reference is $R_{\chi}$ which does not coincide with $R_{x}$; hence, the mapping $\boldsymbol{\Phi}$ is constantly needed to solve the continuity and equilibrium equations. In order to simplify the mapping procedures, the time derivatives are kept in the referential form. Substituting (13) into the Eulerian form of the continuity and equilibrium equations yields 


$$
\begin{aligned}
& \left.\frac{\partial \rho}{\partial t}\right|_{\chi}+c_{\imath} \frac{\partial \rho}{\partial x_{i}}+\rho \frac{\partial v_{i}}{\partial x_{i}}=0 \\
& \left.\rho \frac{\partial v_{i}}{\partial t}\right|_{x}+\rho c_{j} \frac{\partial v_{i}}{\partial x_{j}}=\frac{\partial \sigma_{i j}}{\partial x_{j}}+\rho g_{i}
\end{aligned}
$$

The derivation of the above equations from (20) is given in Appendix A.

The origin of (21) and their similarity to the Eulerian equations have induced some authors [2] to name this method the "quasi Eulerian" description. When implementing (21) for path-dependent materials, it is important to remember the updating difficulties shown in Section 2.2.

In the subsequent development, the viscous fluid is assumed to be isothermal and barotropic (i.e. $F(P, \rho)=0$ ) and that $\partial P / \partial \rho=B / \rho$, with $B$ and $P$ being the fluid bulk modulus and pressure, respectively. The continuity equation (21a) may be rewritten as [35]

$$
\left.\frac{1}{B} \frac{\partial P}{\partial t}\right|_{x}+\frac{\partial v_{i}}{\partial x_{i}}=0 \text { in } R_{x}
$$

\section{or, by introducing}

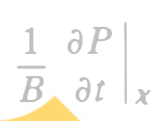

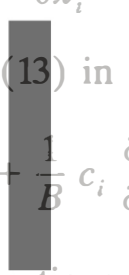

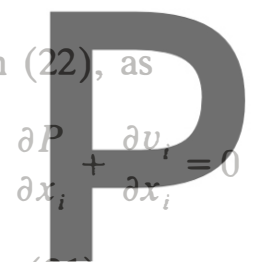

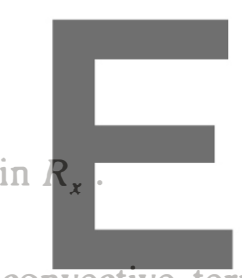

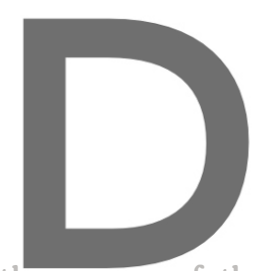

thus,

REMARK 3.1. Equations (21) present a convective term; th Eulerian formulation is still present in the ALE method. Nevertheless, the possibility of

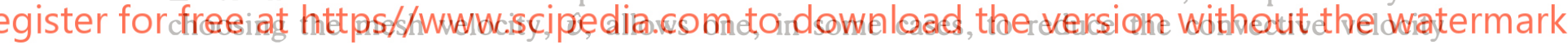
enough to circumvent the associated numerical difficulties.

\subsection{Viscous free surface flow problem}

The object here is to find the velocity and pressure fields satisfying (21b) and (22) and certain specified boundary conditions. It is required that

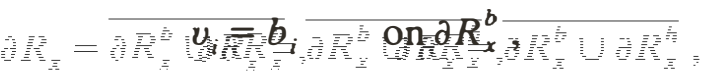

$$
\begin{aligned}
& \text { 青=- }
\end{aligned}
$$

where $\boldsymbol{b}$ and $\boldsymbol{h}$ are the prescribed boundary velocities and tractions, respectively; $\boldsymbol{n}$ is the outward normal to $\partial R_{x}$, and $\partial R_{x}$ is the piecewise smooth boundary of the spatial domain, $R_{x}$. Suppose that $\partial R_{x}$ admits the following decomposition:

with $\partial R_{x}^{b}$ and $\partial R_{x}^{h}$ being subsets of $\partial R_{x}$. The superimposed bar in (25a) represents set closure and $\emptyset$ in (25b) symbolizes the empty set. 
Table 1

Some generalized Newtonian fluid models

\section{Model I ID viscosity}

Newtonian

Power law $\mu_{0}=$ constant

Truncated

Power Law

$$
\mu=m \dot{\gamma}^{n-1}
$$$$
\begin{array}{ll}
\mu=\mu_{0} & \dot{\gamma} \leqslant \dot{\gamma}_{0} \\
\mu=\mu_{0}\left(\dot{\gamma} / \dot{\gamma}_{0}\right)^{n-1} & \dot{\gamma} \geqslant \dot{\gamma}_{0}
\end{array}
$$

3D generalization

Carreau

$$
\mu^{\mu_{x}} \quad \mu_{x}=\left[1+(\lambda \dot{\gamma})^{2}\right]^{(n-1) / 2}
$$

$$
\sigma^{\prime}=2 \mu_{0} D^{\prime}
$$$$
\boldsymbol{\sigma}^{\prime}=2 m\left(\sqrt{2} \overline{\left.\operatorname{tr}\left(D^{\prime 2}\right)\right)^{n-1} D^{\prime}}\right.
$$$$
\boldsymbol{\sigma}^{\prime}=2 \mu_{0} D^{\prime} \quad \sqrt{2 \operatorname{tr}\left(D^{\prime 2}\right)} \leqslant \dot{\gamma}_{0}
$$$$
\boldsymbol{\sigma}^{\prime}=2 \mu_{0}\left(\sqrt{ } 2 \operatorname{tr}\left(\boldsymbol{D}^{\prime 2}\right) / \dot{\gamma}_{0}\right)^{n-1} \quad D^{\prime} \leqslant \dot{\gamma}_{0}
$$

\section{$\sigma^{\prime}=2\left(\mu_{0} \quad \mu_{x}\right)\left[\left(1+2 \lambda^{2} \operatorname{tr}\left(D^{\prime 2}\right)\right]^{(n-1) / 2} D^{\prime}+2 \mu_{\alpha} D\right.$}

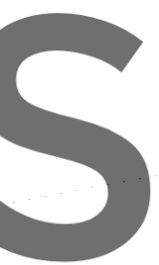

$\mu=\infty \quad \tau \leqslant \tau_{0}$

$\mu=\mu_{p}+\tau_{0} / \dot{\gamma} \quad \tau \geqslant \tau_{0}$

$\mu=\infty \quad \tau \leqslant \tau_{0}$

$\mu=m \dot{\gamma}^{n-1}+\tau_{0} l \dot{\gamma} \quad \tau \geqslant \tau_{0}$

\section{$D^{\prime}=0 \quad \frac{1}{2} \operatorname{tr}\left(\sigma^{\prime 2}\right) \leqslant \tau_{0}^{2}$}

$\sigma^{\prime}=2 \mu_{\mathrm{p}}\left[1+\tau_{0} / \sqrt{2 \operatorname{tr}\left(D^{\prime 2}\right)}\right] \quad D^{\prime} \geqslant \tau_{0}^{2}$

$D^{\prime}=0 \quad \frac{1}{2} \operatorname{tr}\left(\sigma^{\prime 2}\right) \leqslant \tau_{0}^{2}$

$\sigma^{\prime}=2 m\left(\sqrt{ } 2 \operatorname{tr}\left(D^{\prime 2}\right)\right)^{(n-1)} D^{\prime}$

Bulkiey

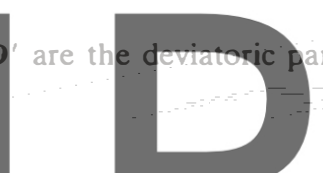

The problem is $n$ between the Cauchy

given. In Table 1 several generalized Newtonian models
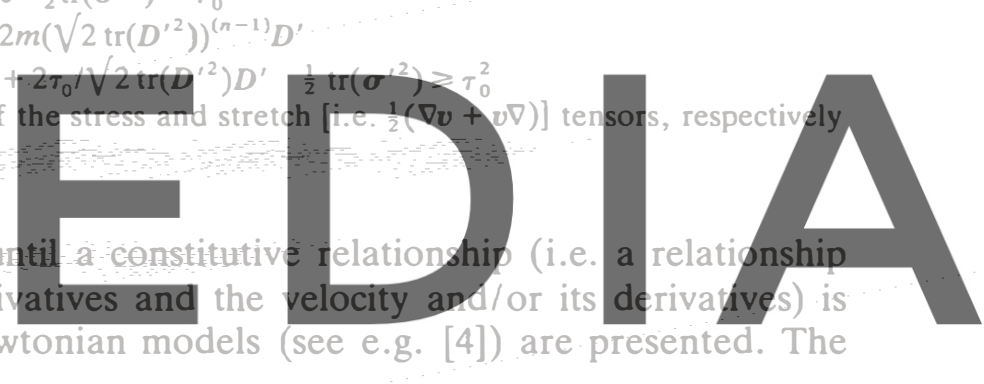

see e.g.

free at https//www.scipedia.col

$$
\dot{\gamma}_{i j}=\frac{\partial v_{\imath}}{\partial x_{j}}+\frac{\partial v_{j}}{\partial x_{\imath}} \text { and }
$$

where $\mu$ is the dynamic viscosity which is shearrate-dependent. The finite element method presented here is independent of the particular generalized Newtonian model chosen.

\section{Automatic rezoning}

\subsection{Introduction}

The possibility of arbitrarily moving the mesh in the ALE description is very interesting. At the same time, the moving boundaries (which are material surfaces) can be tracked with the accuracy characteristic of the Lagrangian methods and the mesh can conserve its regularity to avoid element entanglement. However, this requires that an efficient algorithm be supplied for updating the mesh displacements $(\hat{\boldsymbol{d}})$, velocities $(\hat{\boldsymbol{v}})$, and accelerations $(\hat{\boldsymbol{a}})$, principally on the moving boundaries. Usually the rezoning techniques are based on heuristic developments and only apply to the particular problems for which they were implemented.

The reference frame is fixed, but its movement with respect to the laboratory or the 
continuum is arbitrary, that is, the particle velocity viewed from the reference, $w$, and the mesh velocity, $\hat{\boldsymbol{v}}$, are chosen arbitrarily. However, these two velocities are interrelated by (10); hence, once one of them is determined, the other is automatically fixed. It is important to note that, if $\hat{\boldsymbol{v}}$ is given, $\hat{\boldsymbol{d}}$ and $\hat{\boldsymbol{a}}$ can be computed using difference formulas and there is no need to evaluate $\boldsymbol{w}$. On the other hand, if $\hat{\boldsymbol{v}}$ is unknown but $\boldsymbol{w}$ is given, (10) must be solved to evaluate $\hat{\boldsymbol{v}}$ before updating the mesh. Finally, mixed reference velocities can be given (i.e. $\hat{\boldsymbol{v}}$ can be prescribed in some spatial direction(s) and $w$ in the other(s)). It is obvious that finding the 'best' choice for these velocities and an algorithm for updating the mesh constitutes one of the major problems with the ALE description. Depending on which velocity $(\hat{\boldsymbol{v}}, \boldsymbol{w}$, or mixed) is prescribed, three different cases may be studied.

\subsection{Mesh motion prescribed a priori}

The case where $\hat{v}$ is given corresponds to an analysis where the domain boundaries are known at every instant. Liu et al. [29] used a constant mesh velocity to reduce the transport phenomenon in a pressure wave propagation problem, while Liu et al. [32] used simple ad-hoc formulae to increase the density of elements where unknown functions vary abruptly. When the material boundaries of the fluid domain have a known motion, the mesh movement along this boundary is prescribed priori The rigi
in [18] falls into this type of A problem
4.3. Lagrange-Euler matrix method
The case where w is arbitrarily defined
Hughes et al. [22]. Let $w$ be
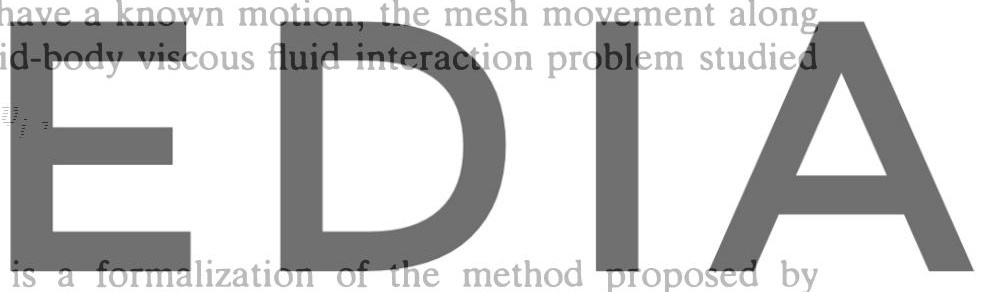
gister for free at https//www.scipedia.com to download the version without the watermark

where $\delta_{i j}$ is the Kroneker delta and $\left[\alpha_{i j}\right]$ is the Lagrange Euler parameter matrix such that $\alpha_{i j}=0$ if $i \neq j$ and $\alpha_{i i}$ is real (underlined indexes meaning no sum on them). In general, the $\alpha$ 's are spatial and time dependent; however they are usually taken as time independent. Equation (27) imposes the condition that $w$ is a linear function of the material velocity and it was chosen because, if $\alpha_{i j}=\delta_{i j}, \boldsymbol{w}=\mathbf{0}$ and the Lagrangian description is obtained, whereas, if $\alpha_{i j}=0, w=v$ and the Eulerian formulation is used. The Lagrange-Euler matrix needs to be given once and for all at each grid point. It is important to notice that (27) presents some disadvantages; for instance, while $\hat{\boldsymbol{v}}$ has a clear physical interpretation (i.e. the mesh velocity), $\boldsymbol{w}$ is much more difficult to visualize (except perpendicular to material surfaces where it is identically zero) and therefore it is very difficult to maintain regular shaped elements inside the fluid domain by just prescribing the $\alpha$ 's. Because of this important drawback the mixed formulation is introduced in the next section.

Since $\boldsymbol{w}$ is defined by (27), the other velocities are determined by (12) and (10), which become, respectively, 
A. Hueria, W.K. Liu, Viscous flow with large free surface motion

and

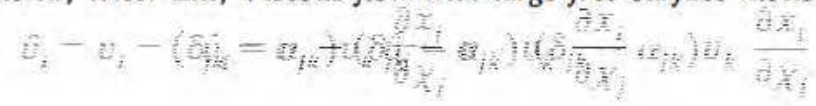

These latter equations must be verified in the referential domain and along its boundaries. Substituting ( $9 \mathrm{~b})$ into (29) yields a basic equation for mesh rezoning

$$
\left.\frac{\partial x_{i}}{\partial t}\right|_{x}+\left(\begin{array}{ll}
\delta_{j k} & \alpha_{j k}
\end{array}\right) v_{k} \frac{\partial x_{i}}{\partial x_{j}} \quad v_{i}=0 .
$$

REMARK 4.1. Equation (30) differs only in its last term from the one proposed by Hughes et al. [22]. This difference is not noticeable if the Lagrange-Euler parameters are chosen equal to zero or one. Moreover, (30) includes the Jacobian matrix (i.e, $\left.\partial x_{i} / \partial \chi_{j}\right)$ that is missing in the formulation of Liu and Ma [35]. Finally, (30) models a pure convection process; hence, the classic numerical difficulties associated with non-selfadjoint equations are expected.

Equation (30) and therefore the ALE technique based on the Lagrange Euler parameters is very useful in problem, such as the propagation of long waves ('tsunamis') and, in generah in every free surface flow where the free surface maty be written as $x_{3 s}=x_{3 s}\left(x_{1}, x_{2}, t\right)$, wi h an Eulerian description used in the $\boldsymbol{y}_{1}$ - and $\boldsymbol{r}_{2}$-directions (i. free surface is defined by onespatial coordinate which is function of the matrix has only one nonzero term, $\alpha_{33}$ (usualiy equal to 1 ) $(30)$ is

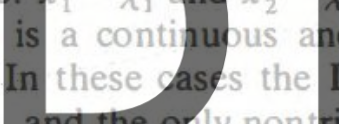

D

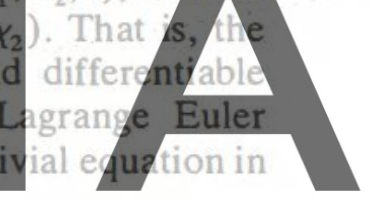

egister for free at https//www.scipedia.com to download the version without the watermark

The above equation is easily recognized as the kinematic equation of the surface and may be

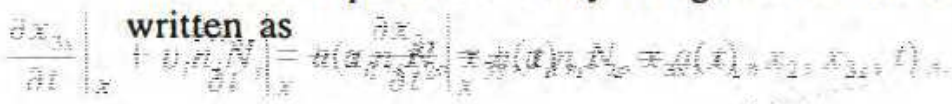

1.4. where $\boldsymbol{n}$ is the exterior unit normal to the surface of components:

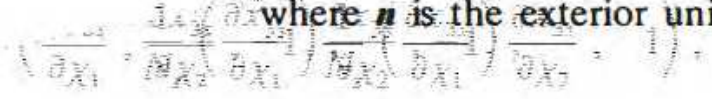

with $N_{s}$ as its norm

$$
\begin{aligned}
N_{s} & =\left[1+\left(\frac{\partial x_{3 s}}{\partial \chi_{1}}\right)^{2}+\left(\frac{\partial x_{3 s}}{\partial \chi_{2}}\right)^{2}\right]^{1 / 2} \\
& =\left[1+\left(\frac{\partial x_{3 s}}{\partial x_{1}}\right)^{2}+\left(\frac{\partial x_{3 s}}{\partial x_{2}}\right)^{2}\right]^{1 / 2},
\end{aligned}
$$




\section{A. Huerta, W.K. Liu Viscous flow with large free surface motion}

and $a\left(x_{1}, x_{2}, x_{3 s}, t\right)$ is the so-called accumulation rate function expressing the gain or loss of mass under the free surface [25], it is deduced after comparing (31) and (32), and it is expressed as

$$
a\left(x_{1}, x_{2}, x_{3 s}, t\right)=\left(\alpha_{33}-1\right) v_{3} \frac{\partial x_{3 s}}{\partial \chi_{3}}=w_{3} \frac{\partial x_{3 s}}{\partial \chi_{3}} .
$$

The free surface is a material surface (in isothermal processes); along this boundary the accumulation rate must be zero, and consequently $\alpha_{33}$ has to be taken equal to one. This can also be concluded by noticing that no particles can cross the free surface; hence, $w_{3}$ must be zero. Although (30) can be applied to problems where $x_{1}$ and/or $x_{2}$ are non-Eulerian by prescribing nonzero $\alpha$ 's in these directions, controlling the element shapes is very difficult by adjusting the $\alpha$ 's.

\subsection{Mixed formulation}

Noticing the restrictions of the $\alpha$ 's scheme, a mixed formulation is proposed for the resolution of (10). One of the goals of the ALE method is the accurate mapping of the moving boundaries which are usually material surfaces. Hence, along these surfaces $\boldsymbol{w} \cdot \boldsymbol{n}=0$, where $\boldsymbol{n}$ is the exterior normal. The other goal of the ALE technique is to avoid element entanglement and this is better achieved, once the boundaries are known, by prescribing the mesh displacements (through potential equations, for instance) or velocities, because both $\hat{d}$ and $\hat{v}$ govern directly the element shape. Therefore, one should be able to prescribe $\boldsymbol{w} \cdot \boldsymbol{n}=0$ along the domain boundaries while defining the $\hat{d}$ 's or $\hat{v}$ 's in the interior.

The system of differential equations defined in $(10)$ has to be solved along the moving boundaries. Notice first that solving for $w_{i}$ in terms of $\left(v_{i}-\hat{v}_{i}\right)$, equation (10) can be rewritten as

or

$$
\left.\partial x_{i}\right|_{x}-v_{i}-\sum_{\substack{j=1 \\ j \neq i}}^{N S D} v_{j}-\hat{v}_{j} \hat{J}^{\underline{u}}=-\frac{\hat{\jmath}}{\hat{J}^{\underline{i}}} w_{\underline{i}},
$$

where $\hat{j}$ is the Jacobian already defined in $(20 \mathrm{c}), \hat{\jmath}^{i j}$ is the cofactor (i.e. signed minor determinant) of the element $\partial x_{i} / \partial \chi_{j}$ in the Jacobian matrix, and NSD is the number of space dimensions. Notice that the cofactor $\hat{J}^{\|}$appears in the denominator to account for the motion of the mesh in the plane perpendicular to $\chi_{i}$ because (35) are verified in the reference domain $R_{x}$, not in the actual deformed domain $R_{x}$.

For simplification purposes and without any loss of generality, assume that the moving boundaries are perpendicular to one coordinate axis in the reference domain. Let the free surface be perpendicular to $\chi_{3}$, the first two equations in (35) are trivial because in the direction of $\chi_{1}$ and $\chi_{2}$ the mesh velocity is prescribed and therefore the mesh motion is known, but the third one must be solved for $\hat{v}_{3}$, given $w_{3}, \hat{v}_{1}$, and $\hat{v}_{2}$, it may be written explicitly as

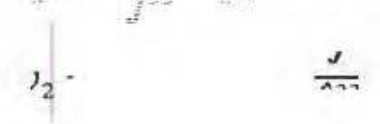


or

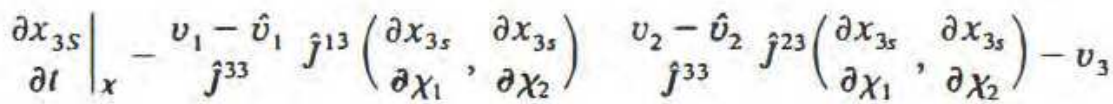

$$
\begin{aligned}
& \left.=\begin{array}{c}
-w_{3} \\
\hat{J}^{33}
\end{array}\right\}\left(\begin{array}{ll}
\partial x_{3 s} & \partial x_{3 s} \\
\partial \chi_{1} & \partial \chi_{2}
\end{array}\right),
\end{aligned}
$$

where $\hat{v}_{3}$ has been substituted by $\partial x_{3 s} /\left.\partial t\right|_{\chi} ; \hat{J}^{13}, \hat{J}^{23}$, and the Jacobian $\hat{J}$ are functions of $\partial x_{3 s} / \partial \chi_{1}$ and $\partial x_{3 s} / \partial \chi_{2} ; \hat{J}^{33}$ is not dependent on $x_{3 s}$; and $x_{3 s}$ is the free surface equation. In (37) $x_{3 s}$ is the unknown function, while $\hat{v}_{1}, \hat{v}_{2}$, and $w_{3}$ are known. If $\hat{v}_{1}=\hat{v}_{2}=0$ (i.e. the Eulerian description is used in $\chi_{1}$ and $\chi_{2}$ ), the kinematic surface equation (32) is again obtained. However, with the mixed formulation $\hat{v}_{1}$ and $\hat{v}_{2}$ can be prescribed (as a percentage of the wave celerity, for instance) and therefore better numerical results are obtained than by defining in (31) $\alpha_{11}$ and $\alpha_{22}$, whose physical interpretation is much more obscure.

It is important to notice that with the mixed formulation the mesh updating equations (35) or (36) are solved, exclusively, along the moving boundaries; whereas in the Lagrange Euler matrix method the mesh updating equations (30) are solved over the complete domain. As remarked previously, in the interior of the domain several techniques are available for the mesh rezoning, for instance, potential equations may be defined and solved or, more simply, the mesh velocities prescribed directly as a percentage of the mesh velocities at the boundaries. An example of this latter technique is applied to the dam-break and largeamplitude sloshing problems studied here.

\section{Finite element formulation}

At this point the conservation equations (21b) and (22), the boundary conditions (24), the constitutive relationship (26), and the mesh updating equations (30) or (35b) define the boundary value problem that needs to be solved. In order to apply the finite element method (FEM) to this problem, all of the above-mentioned differential equations must be transformed into equivalent integral equations. This transformation is based in the weighted residual theory, which can be formulated in different manners. Here, the Petrov-Galerkin formulation is used for the equilibrium and mesh updating equations, while the Galerkin method is applied to the-continuity equation. Furthermore, a pressure-velocity $(P-v)$ formulation is implemented for numerical efficiency and accuracy. Constant pressure elements are used; thus, both weight and trial pressure functions are constant inside the element and discontinuous across interelement boundaries. The pressure weighting function is denoted as $\delta P$, and the integral equation associated with the continuity equation (22) is

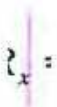

where the spatial domain, $R_{x}$, is discretized into element subdomains, $R_{x}^{e}$, and $\Sigma_{e}$ symbolizes the sum over all elements.

For the equilibrium equation the streamline-upwind/Petrov-Galerkin formulation requires discontinuous weighting functions of the form 


$$
\delta v=\delta \omega+\partial p
$$

where $\delta \omega$ is continuous in $R_{x}$ and $\delta p$ is the discontinuous streamline upwind perturbation; $\delta p$ is assumed smooth in the element interior. It can be shown [6] that weighting the convective term with $\delta v$, defined by (39), is equivalent to introducing an added artificial diffusivity. However, $\delta \boldsymbol{v}$ must be applied to all terms in the equation; otherwise, an inconsistent weighted residual formulation would result. The variational equation resulting from (21b) is

$$
\begin{aligned}
& \int_{R_{x}} \delta \omega_{t}\left[\left.\rho \frac{\partial v_{i}}{\partial t}\right|_{x}+\rho c_{j} \frac{\partial v_{i}}{\partial x_{j}}-\frac{\partial \sigma_{i j}}{\partial x_{j}}-\rho g_{i}\right] \mathrm{d} R_{x} \\
& +\sum_{e} \int_{R_{x}^{e}} \delta p_{i}\left[\left.\rho \frac{\partial v_{i}}{\partial t}\right|_{x}+\rho c_{j} \frac{\partial v_{i}}{\partial x_{j}}-\frac{\partial \sigma_{i j}}{\partial x_{j}}-\rho g_{\imath}\right] \mathrm{d} R_{x}=0,
\end{aligned}
$$

which, together with the constitutive equation (26) and the natural boundary conditions (24b) yields

$$
\begin{aligned}
& \int_{R_{\nexists}^{e}}
\end{aligned}
$$

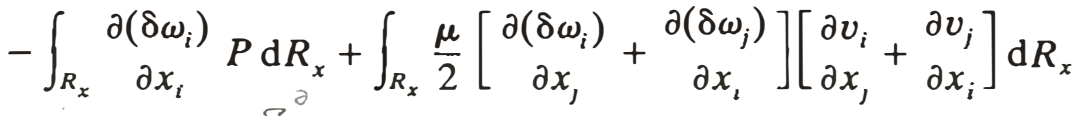

$$
\begin{aligned}
& -\sum_{e} \int_{R_{x}^{e}} \partial p_{i} \partial \sigma_{i j}^{c} \mathrm{~d} R_{x}-\sum_{e} \int_{R_{x}^{e}} \delta v_{i} \rho g_{\imath} \mathrm{d} R_{x}-\int_{\partial R_{x}^{h}} \delta \omega_{i} h_{i} \mathrm{~d} S=0 .
\end{aligned}
$$

The influence of the perturbation, $\delta p$, on the diffusive term, $\partial \sigma_{i j} / \partial x_{j}$, is neglected pursuant to the extensive discussion given by Hughes and Brooks [20]. Under this circumstance and from (41) one can note that the perturbation affects only the fluid mass, the convective, and the body force terms, and does not influence the viscous, the pressure, and the boundary force terms.

Finally, the integral equation associated with the mesh updating formulas (30) or (35b) is obtained using again the streamline-upwind/Petrov-Galerkin method, where the weighting functions, $\delta x$, are considered to be composed of both the continuous interpolation functions and the perturbation functions. However, these equations are of the pure convection type; therefore, following the method presented by Hughes and Tezduyar [24], (30) becomes

$$
\text { - di } n_{x},
$$

and (35b) yields

$$
\left.\int_{R_{\chi}} \delta x_{\imath} \frac{\partial x_{i}}{\partial t}\right|_{\chi} \mathrm{d} R_{\chi}-\int_{R_{\chi}} \delta x_{i} \sum_{\substack{j=1 \\ j \neq i}}^{\mathrm{NSD}} \frac{v_{j}-\hat{v}_{j}}{\hat{J}^{\underline{\underline{I}}}} \hat{J}^{j \underline{i}} \mathrm{~d} R_{\chi}-\int_{R_{\chi}} \delta x_{i}\left(v_{i}-\frac{\hat{J}}{\hat{J}^{i \underline{i}}} w_{i}\right) \mathrm{d} R_{\chi}=0 .
$$




\section{A. Huera, W.K. Lill, Viscous flow with large free surface motion}

A more detailed description of $\delta x$ for each rezoning technique may be found in Appendix $\mathrm{B}$. In general, (42b) can be used to update the finite element mesh but to enhance the computational efficiency, (42b) is used only on the free surface while simple techniques are implemented in the interior of the domain, see for instance the flow of a fluid over a dry bed or the sloshing problems presented here.

REMARK 5.1. Both the continuity and equilibrium equations must be mapped into the spatial domain for integration; see (38) and (41). However, the mesh updating equations are already written in referential form and thus the associated variational equations are integrated over the fixed reference; see (42).

$R E M A R K$ 5.2. The discontinuous streamline upwind perturbation function, $\delta p$, is written as [6]

$$
\delta p_{i} \quad \begin{gathered}
\partial\left(\delta \omega_{i}\right) \\
\partial x_{j}
\end{gathered} \frac{c_{j} k}{\|c\|^{2}}
$$

where the artificial diffusivity, $\bar{k}$, may be defined using a spatial eriterion $[6]$

or a temporal criterion [24]

$$
\bar{\eta} \operatorname{cotgh}\left(\alpha_{\eta}\right)-1 / \alpha_{\eta}, \quad \alpha_{\eta} \frac{c_{\eta} h_{\eta} \rho}{2 \mu} .
$$

\section{Matrix equations and predictor-multicorrector algorithm}

The spatial discretization of the integral equations (38), (41), and (42) leads to the following system of partial differential equations:

$$
\begin{aligned}
& M a+\eta(v)+K_{\mu} v-G P=f^{\mathrm{ext}}, \\
& \hat{M} \hat{v}+\hat{\eta}(x)-\hat{M} v \quad 0,
\end{aligned}
$$


where the superscript ' $\mathrm{t}$ ' denotes matrix transpose; $\boldsymbol{M}^{P}, \boldsymbol{M}$, and $\hat{\boldsymbol{M}}$ are the generalized mass matrices for pressure, velocity, and mesh velocity, respectively; $\boldsymbol{\eta}^{P}, \boldsymbol{\eta}$, and $\hat{\boldsymbol{\eta}}$ are the generalized convective terms for pressure, velocity, and mesh velocity; $f^{\text {ext }}$ is the total external load vector applied on the fluid; $\boldsymbol{K}_{\mu}$ is the fluid viscosity matrix; $\boldsymbol{G}$ is the divergence operator matrix; $\boldsymbol{v}, \boldsymbol{P}$, and $\hat{\boldsymbol{v}}$ are the vectors of unknown nodal values for velocity, pressure, and mesh velocity, respectively; and $\dot{\boldsymbol{P}}$ and $\boldsymbol{a}$ are the time derivative of the pressure and the material velocity holding the reference fixed. The definitions for these matrices and vectors are given in Appendix B. Note that (46c) is the matrix form of (42b) if the updating surface is a material surface, which usually is the case.

The predictor-multicorrector algorithm presented by Liu and Gvildys [33] is used to solve (46a) and (46b). Once the pressure and velocity fields are computed, (46c) is solved again using a predictor-multicorrector algorithm. This implies that an important numerical overburden is created, because the loop for solving (46c) is inside the main loop for computing the pressure and particle velocity fields (i.e. (46a) and (46b)). However, it is important to notice that in order to suppress any 'diffusion' between Lagrangian and Eulerian nodes (see [22]), the Lobatto integration is employed to obtain (46c), thus $\hat{M}$ is diagonal and the resolution of (46c) is explicit.

Finally, it should be noted that, in order to increase the numerical efficiency of the computer code, variable time stepping can be implemented. The limits for the time increments may be found in [7]. The equilibrium equation for a viscous fluid is of the advection-diffusion type; the time step is determined by the diffusion, advection, and Courant-Friedrichs-Levy (CFL) conditions, with $c$ being the convective velocity. However, for the mesh updating equation, which is a pure advection process, the CFL condition is the only one to be applied with $\left(\delta_{i j}-\alpha_{i j}\right) v_{j}$ as the components of the convective velocity.

REMARK 6.1. For the Lagrange-Euler matrix method with a Galerkin formulation, the particular nature of (46c) allows its simplification to (see Appendix C)

$$
\hat{\boldsymbol{M}} \hat{\boldsymbol{v}}=\hat{\boldsymbol{M}} \boldsymbol{v}-(\boldsymbol{L v}) \boldsymbol{x},
$$

where $\boldsymbol{L}$ is a banded third-order matrix that can be computed after reading the data, stored, and used in every time step, as well as the generalized mass matrix, $\hat{\boldsymbol{M}}$, which is also diagonal; recall that these matrices are obtained after integration over the referential domain (Remark 5.1) which is, by definition, fixed in time. This saves considerable computer time because there is no need to create any matrix for the mesh updating equation at every time step and iteration. The size of $\boldsymbol{l}$ is NMEQ $\times$ MAXMN $\times$ NSD, where NMEQ is the number of mesh equations (a mesh equation exists when, at a given node and spatial dimension, the mesh motion is not prescribed); MAXMN is the maximum difference, for all elements, between two equation numbers corresponding to nodes in the same element; and NSD is the number of spatial dimensions. The size of $\hat{\boldsymbol{M}}$ is simply NMEQ.

\section{Numerical examples}

In this section the ALE formulation is applied to three different engineering problems. The first one is a numerical model of propagation of long waves ('tsunamis') onto a shelf, i.e. the 
reflection, transmission, and propagation of the waves on the shelf. The results are compared to other numerical solutions and to experimental results with good agreement in both cases.

The second example is an attempt to model the breaking of a dam or more generally a non-Newtonian flow with large free surface motion. This problem, which has an approximate solution for an inviscid fluid flowing over a perfect frictionless bed, presents a formidable challenge when this solution is applied to mine tailings embankments. Attempts have been made to study this problem [26] or similar ones [5], but too many assumptions are necessary in both the flow model and the material characterization. Here the finite element method allows a complete integration of the field equations and the use of constitutive models better suited to tailings. Nevertheless, some applications require simp ifications that offer room for subsequent improvement.

In the last example, forced horizontal oscillations are imposed to evaluate the sloshing response of a water pool. The nonlinear treatment of the free surface motion yields good comparisons between the numerical results and experimental tests, and moreover, large wave heights, amounting approximately to $50 \%$ of the depth of the container, are easily modeled.

\subsection{Propagation of long waves-tsunamis}

Tsunamis or tidal waves are long waves (i.e. waves with lengths that are large compared to the depth of water in which they are propagating) usually generated by earthquakes. Figure 2(a) shows a schematic section of the continental slope off the coast of California, and Fig. 2(b) presents a finite element modeling of this problem after scaling by characteristic quantities [13]. Notice that in the dimensionless problem the continental slope may be approximated by a step. Several events may be distinguished in this problem; first, the propagation of the incident wave towards the shelf in a constant-depth ocean; then, the creation of the reflected and transmitted waves when the incident wave hits the step; and finally, the propagation of these new waves in constant-depth domain.

The propagation of a solitary wave over a constant depth is first studied. Shallow water theory [46] is the classical approach to this problem, but Hughes et al. [22] applied the ALE method with good comparisons between both models. Moreover, the ALE technique with two elements in depth was more consistent with the experimental results because the dispersion emanating from the back of the wave in the previous numerical examples was considerably reduced; see Figs. 3 and 4. Here the same problem is studied; that is, the wave is generated by prescribing the displacement history at the left-hand boundary of the fluid domain:

where

$$
d_{1} \frac{\eta}{\kappa}\left[1+\tanh \left(\frac{c \kappa t}{D}-4\right)\right]
$$

$$
\begin{aligned}
& c=\sqrt{ } g(D+\eta) \\
& \kappa=\sqrt{ } 3 \eta / 4 D,
\end{aligned}
$$

$g=1, L=949.095, D=10, \eta=0.86$, and $\Delta t=1.7888$. Along all boundaries except the free surface, perfect sliding is assumed (shear stress equal to zero) because the fluid is inviscid. In this problem both ALE techniques, the Lagrangian-Euler matrix method and the mixed formulation, are equivalent because an Eulerian description is chosen in the horizontal 
A. Huerta, W.K. Liu, Viscous flow with large free surface motion
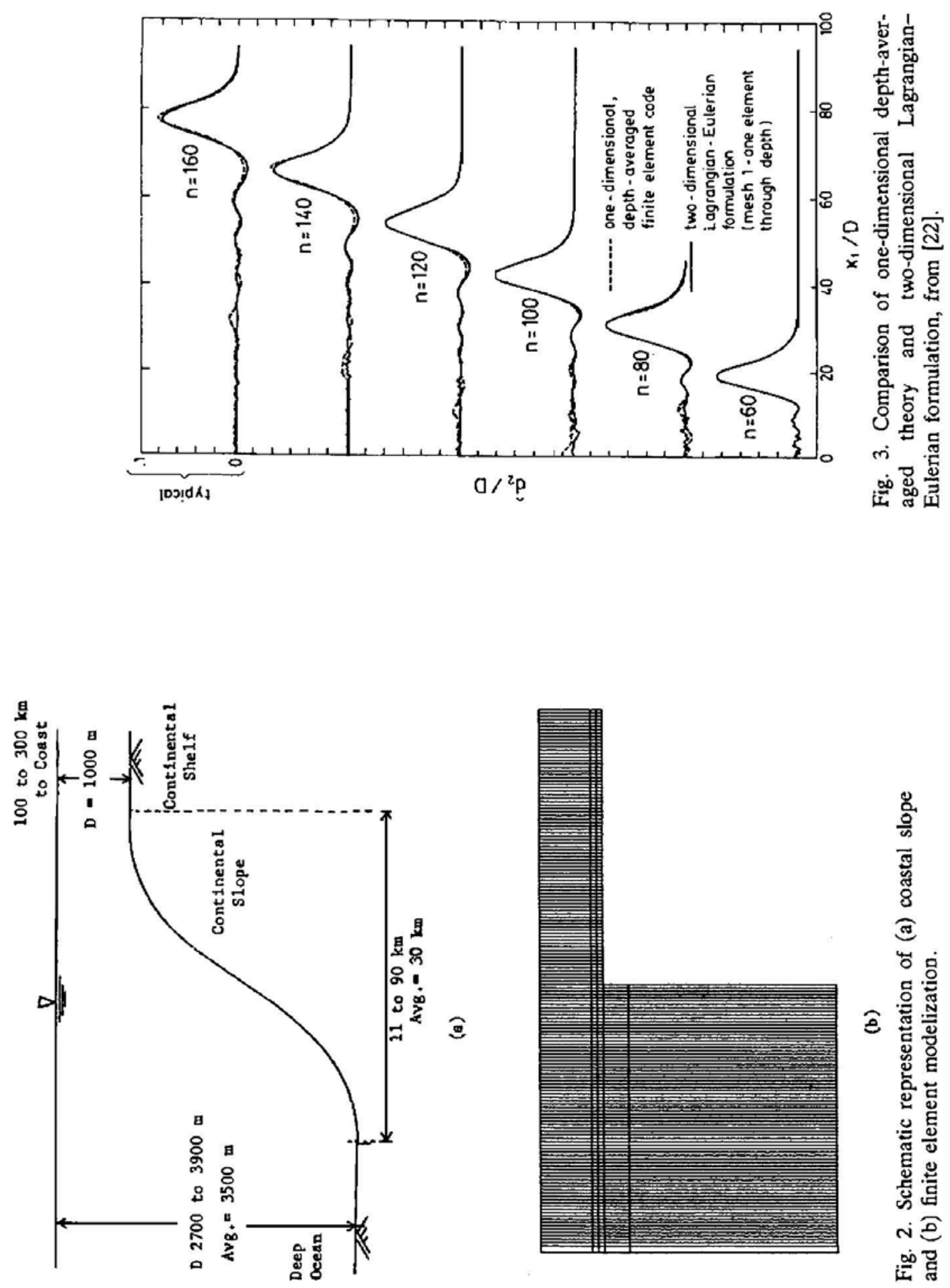
A. Huerla, W.K. Liu, Viscous flow with large free surface motion

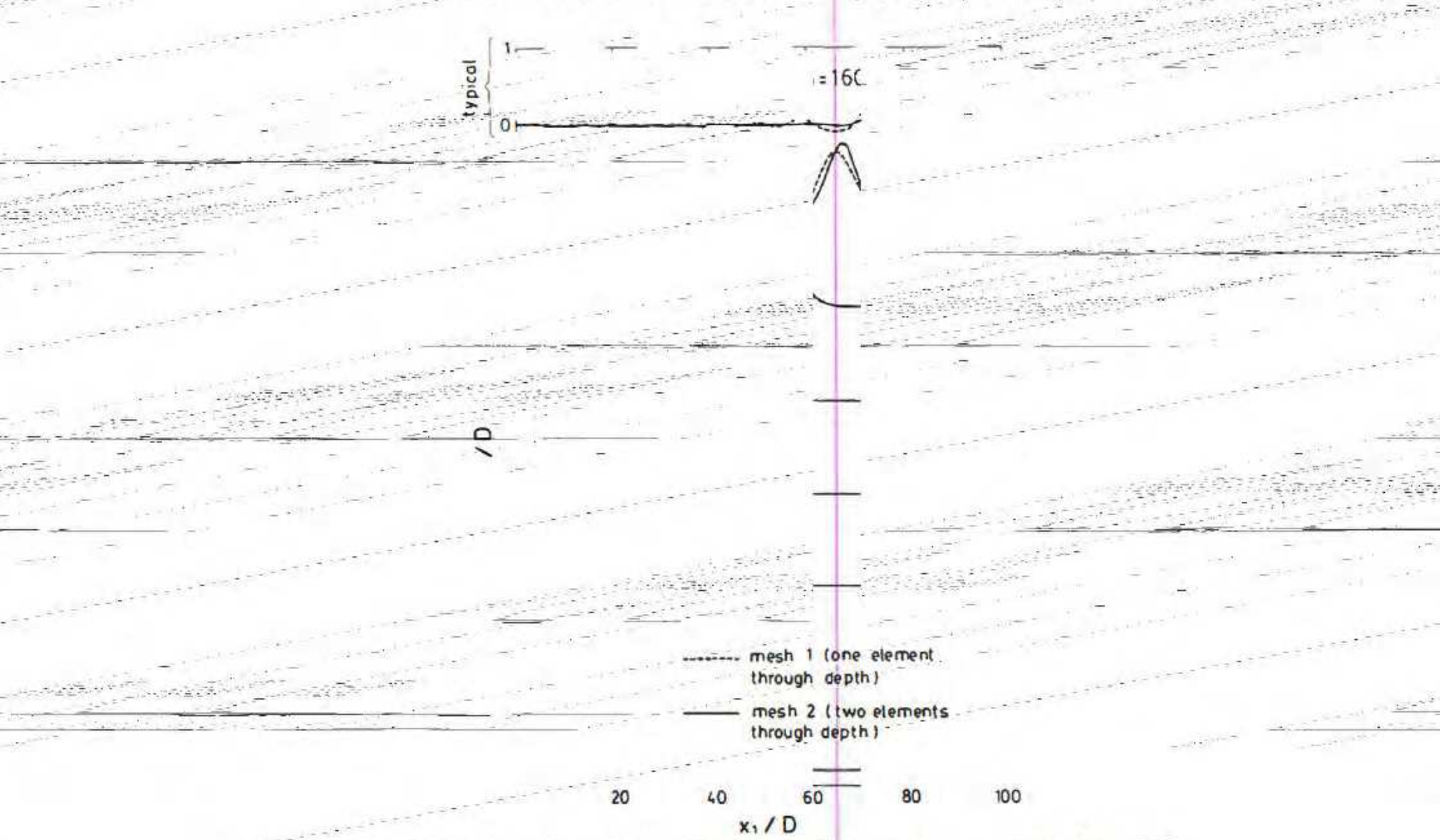

Fig. 4. Comparison of results for one and two elements through depth, from [22].

direction. Figure 5 shows the computed results with one element in depth and a 160 4-node element mesh, as in Hughes et al. [22]. Comparing the results in Fig. 5 with those in Figs. 3 and 4 , it is obvious that the predictor-multicorrector algorithm proposed here with one element in depth and single precision-calculations (32 bits per real $* 4)$, gives better results than shallow water theory or ALE (implicit formulation double precision, 64 bits per real $* 8$ ) with the same number of elements; in fact, the results compare better with the Hughes et al. [22] solution using two elements through depth. The yariation of wave volume during the 320 time steps (the wave travels one tank length, is reflected, and travels back) is $0.02 \%$. It is believed that the improvement obtained with the present formulation (i.e. less dispersion emanating from the back of the wave and less amplitude decay of the wave for the same number of elements) is due to the fact that a $P-v$ formulation models more accurately the fluid incompressibility than the penalty formulation, and that two passes instead of one are used to solve the nonlinear free surface updating equation.

Figure 6 shows the reflection and transmission of the wave generated as before, over a step with a ratio $D_{1} / D_{2}=4$; this value was chosen because it is similar to the shelf ratio off the coast of California. This figure clearly reproduces the creation of the reflected wave and how the transmitted wave changes its shape as it propagates, increasing its amplitude and 
A. Huerta, W.K. Liu, Viscous flow with large free surface motion
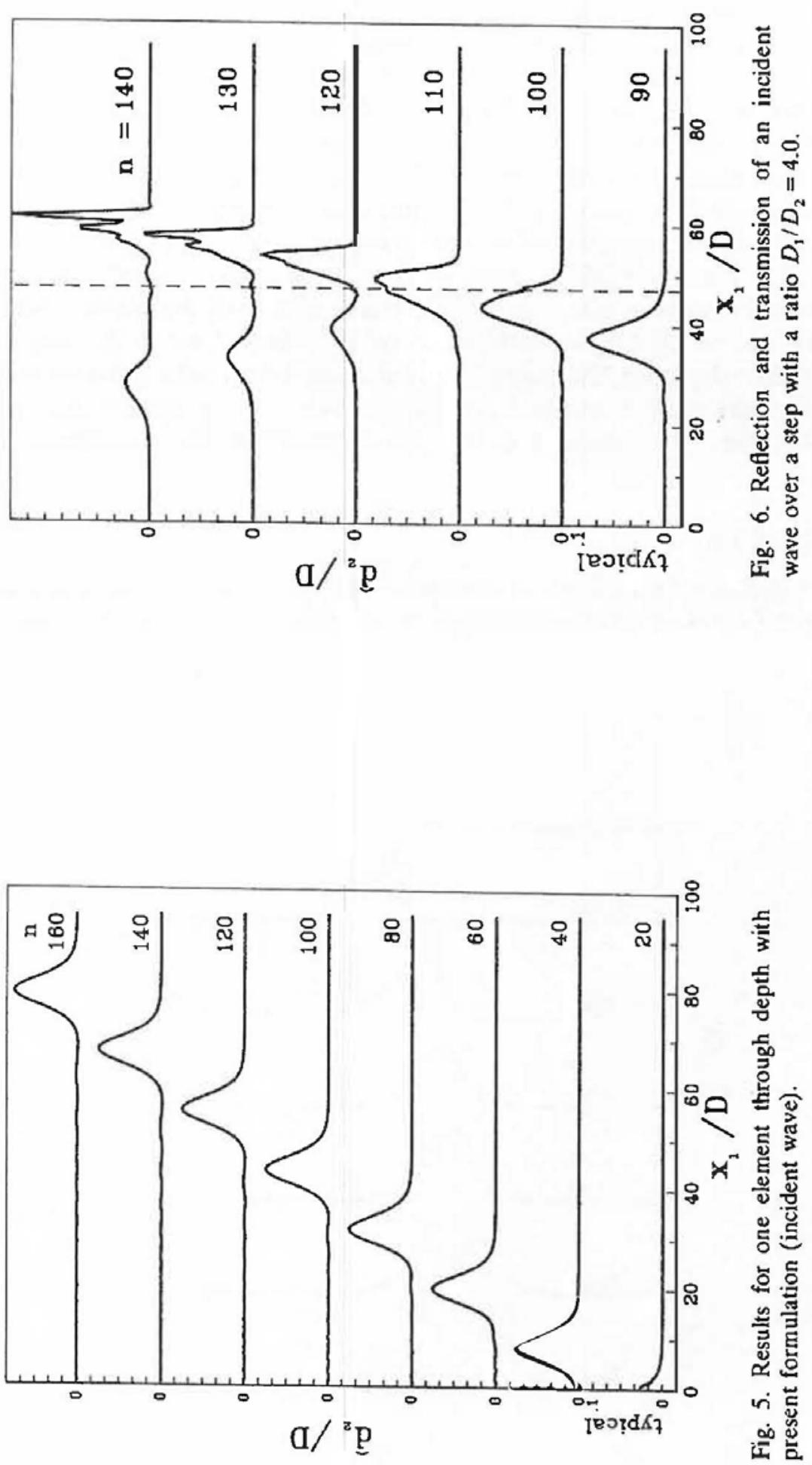


\section{A. Huerta, W.K. Liu, Viscous flow with large free surface motion}

frequency and then transforming into a series of solitary waves. To compare the numerical solution of the step problem with the experimental results of Goring [13], the following parameters were taken: $D_{1}=10, D_{1} / D_{2}=4.65, \eta / D_{1}=0.95$, and $\Delta t=0.8944$. The domain discretization is shown in Fig. 2(b) and it consists of 638 4-node elements and 804 total mesh nodes. Figure 7 shows the time variations of several surface points; the origin is taken at the step. The calculated results compare satisfactorily with the experimental data. Moreover, the theoretical models applied by Goring [13] (i.e. linear nondispersive theory, nonlinear nondispersive theory, and nonlinear dispersive shallow-water theory) predicted at $x_{1} / D_{2} \simeq 8.23$ waves of at least the same amplitude as at $x_{1} / D_{2}=0$, but here a small decrease, consistent with the experimental results, is observed. The full integration of the Navier Stokes equations over depth allows one to model the turbulence on the shelf close to the step, and thus the dissipative effects that decrease the wave amplitude are taken into consideration. Finally, it should be noted that the present method predicts that the wave is transformed into a series of solitary waves, but this phenomenon is less-accentuated in the experiments than in the numerical model.

\subsection{Dam-break problem}

The dam-break problem is a classic in mathematical hydraulics; it was first solved by Ritter in 1892 , by using the method of characteristics to compute the motion of a semi-infinite body

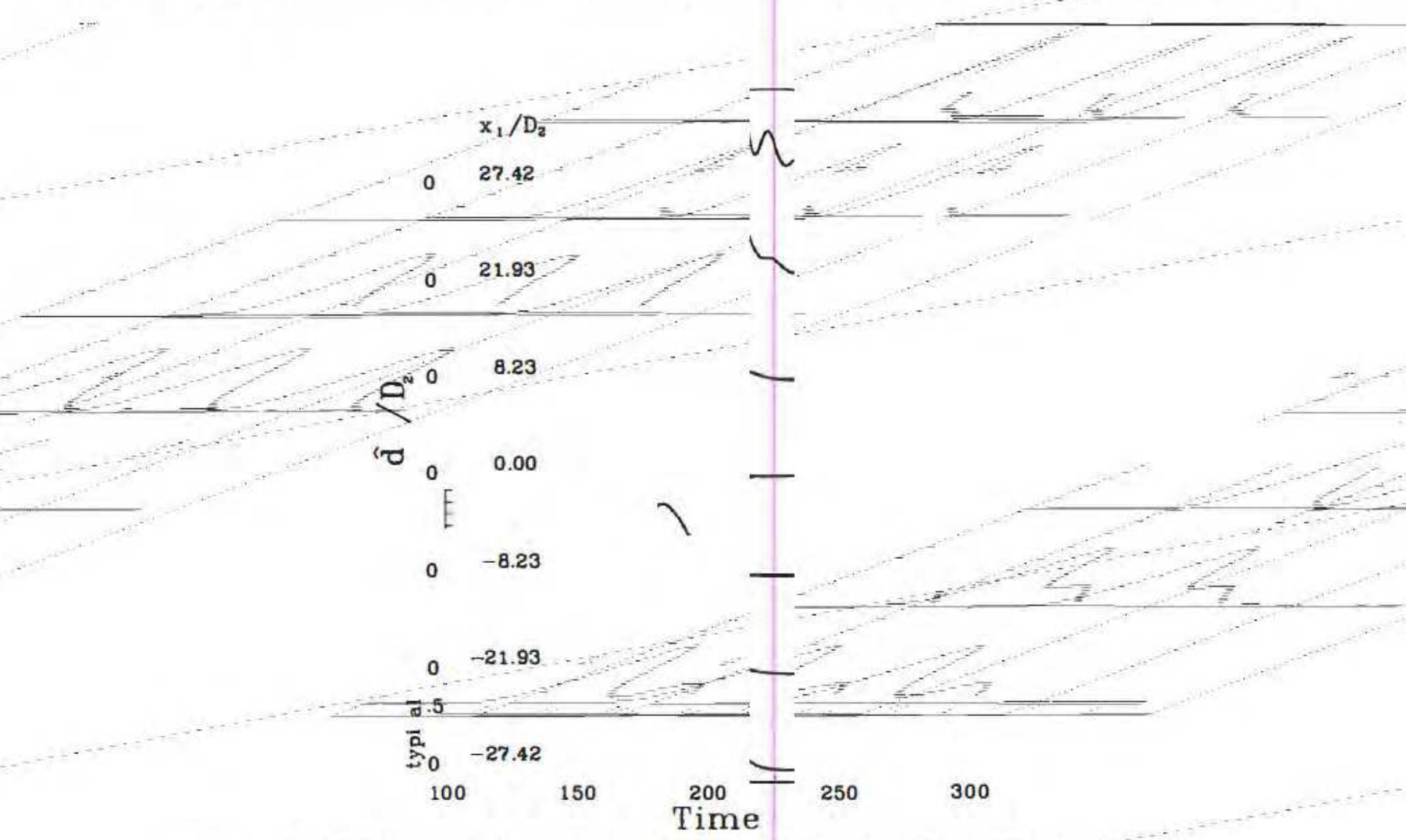

Fig. 7. Free surface oscillations at different locations for $D_{1} / D_{2}=4.65$ and $\eta / D_{1}=0.95$. 
of water, initially at rest, over a horizontal frictionless bed. Because of the differences between Ritter's solution and experimental data, Dressler [12] and Whitham [48] introduced the effect of the bed resistance; the former employed a perturbation technique based on the Chézy empirical resistance constant, while the latter used the Pohlhausen method which is usually applied in boundary layer problems. These solutions were enhanced by numerical techniques which accounted for sloping beds and finite reservoirs $([8,26,45]$ among others). In every case, the downstream boundary condition was solved by introducing a small, but finite, depth of still fluid, by enforcing Whitham's tip solution, or by assuming that the entire tip region moves as a rigid body. However, the most important characteristic of all of the solutions is that they are based on shallow water theory (i.e. the Saint Venant equations; e.g. [46]).

In shallow water theory the rate of energy dissipation is uniquely related to the boundary shear and expressed in the same way as in steady uniform flow. This boundary friction term is used to match analytic and experimental results, and the relationship may be written as

$$
f_{0}+f_{1} \tilde{v}+f_{2} \tilde{v}^{2}
$$

where $\tilde{v}$ is the average velocity in the channel section and $f_{0}, f_{1}$, and $f_{2}$ denote the static, laminar, and turbulent friction, respectively. In classical water flooding analyses, $f_{0}$ and $f_{1}$ are zero, while $f_{2}$ is related to the Chézy coefficient. Jeyapalan [26] studied Newtonian laminar flow by prescribing $f_{0}$ and $f_{2}$ as zero and relating $f_{1}$ to the viscosity; then he extended his analysis to Bingham materials by prescribing $f_{0}$ as a function of the yield stress. Finally, Brugnot and Pochet [5] computed $f_{0}, f_{1}$ and $f_{2}$ experimentally for snow avalanches and concluded that the stop length of the avalanche is highly sensitive to these coefficients. Other assumptions inherent to the Saint Venant equations are that the velocity distribution over a cross-section is essentially uniform and that the streamline curvature is small (i.e. parallel flow and hence the pressure distribution with depth is hydrostatic).

In this study the dam-break problem is solved without the restraints imposed by shallow water theory; two problems are discussed: flow over a still fluid (FSF) and flow over a dry bed (FDB). In both cases the accuracy of the ALE finite element approach is checked by solving the inviscid case, which has an analytical solution in shallow water theory; then, other viscous cases are studied and discussed.

The dimensionless problem is defined by employing the following characteristic dimensions: the length scale is the height of the dam, $H$, over the dry bed or the surface of the downstream still fluid; the characteristic velocity, $\sqrt{g H}$, is chosen to scale velocities; and $\rho g H$ is the pressure scale. The characteristic time is arbitrarily taken as the length scale over the velocity scale, i.e. $\sqrt{H / g}$.

Consequently, if the fluid is Newtonian, the only dimensionless parameter associated with the field equations is the Reynolds number, $\operatorname{Re}=H \sqrt{g} \bar{H} / \nu$, where $\nu$ is the kinematic viscosity. However, this problem is governed by several other parameters besides Re; for instance, the slope of the dam, the height of the still fluid, and the type of non-Newtonian model employed. A complete parametric analysis may be found in Huerta [17], where the influence of the previous physical parameters and other numerical constants is discussed.

Apart from the classical difficulties associated with the resolution of the free surface and the Navier-Stokes equations for incompressible fluids, the dam-break problem presents some 


\subsubsection{Flow over a still fluid}

Along the upstream and downstream boundaries a frictionless condition is assumed, whereas on the bed perfect sliding is only imposed in the inviscid case (for viscous flows the velocities are set equal to zero). In the horizontal direction 41 elements of unit length are usually employed, while in the vertical direction one, three, five, or seven layers are taken, depending on the particular case (see Fig. 9). For the inviscid analysis, $\Delta H=H=1$, as in [36]. In this problem both the Lagrange-Euler matrix method and the mixed formulation are equivalent because an Eulerian description is taken in the horizontal direction; in the vertical direction a Lagrangian description is used along the free surface while an Eulerian description is employed everywhere else.

Figure 10 compares the shallow water solution with the numerical results obtained by the one and three layers of elements meshes. Notice how the full integration of the Navier Stokes equations smoothes the surface wave and slows down the initial motion of the flooding wave (recall that the Saint Venant equations predict a constant wave celerity, $\sqrt{g H}$, from $t=0$ ). No important differences exists between the two discretizations (i.e. one or three elements in depth); both present a smooth downstream surface and a clearly separate peak at the tip of the wave. It is believed that this peak is produced in large part by the sudden change in the
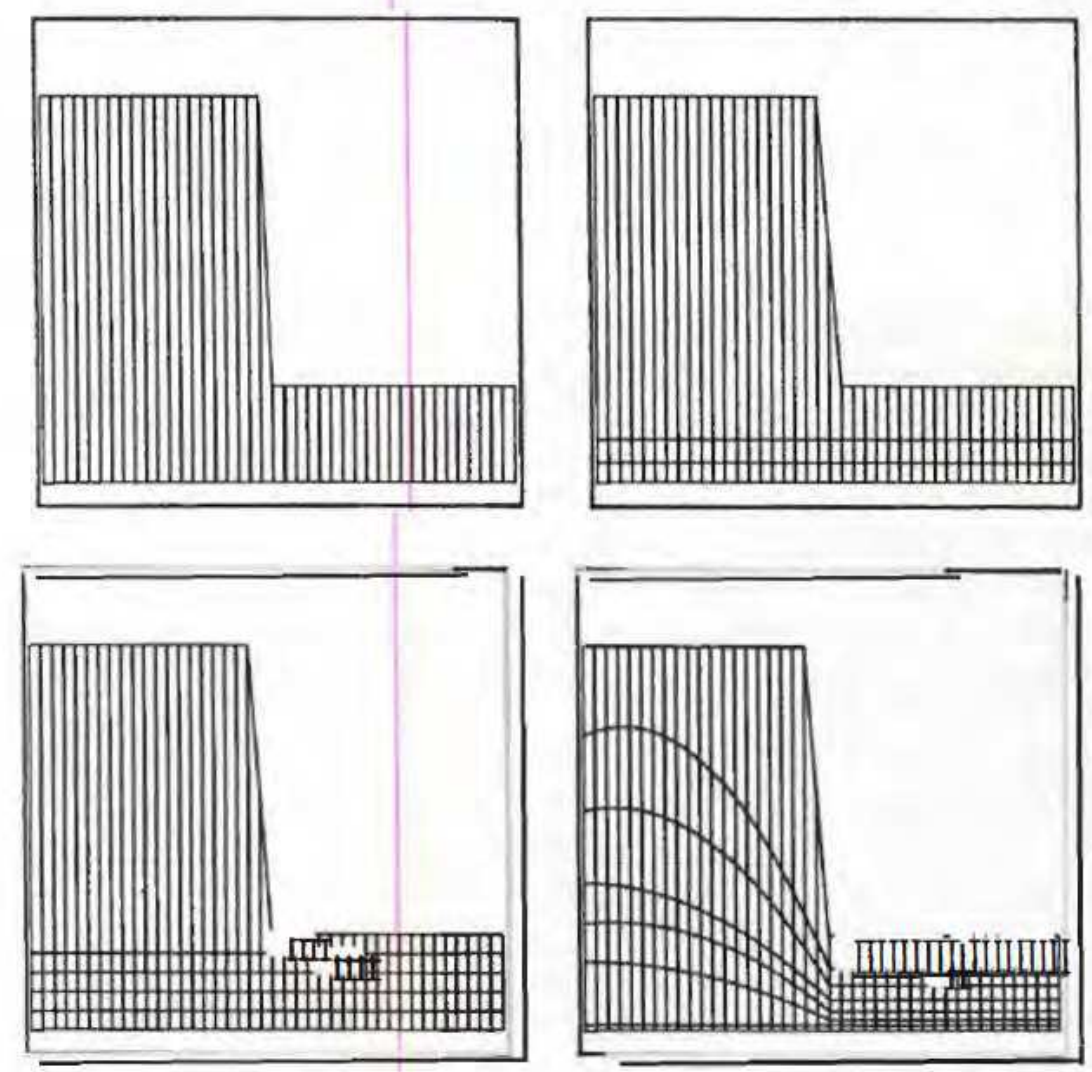

Fig. 9. Mesh discretizations employed. (a) $41 \times 1$, (b) $41 \times 3$, (c) $41 \times 5$, (d) $41 \times 7$. 
A. Huerta, W. K. Liu, Viscous flow with large free surface motion

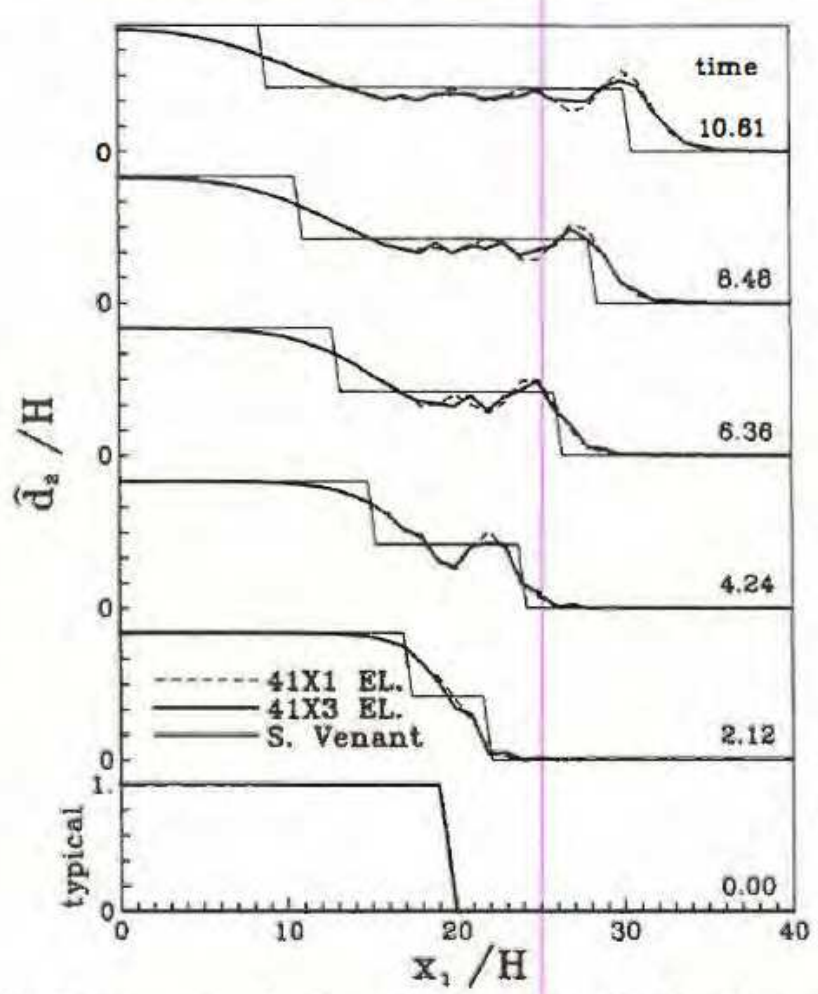

Fig. 10. Comparison of the shallow water theory and Lagrangian-Eulerian formulation for two discretizations (FSF).

vertical component of the particle velocity between still conditions and the arrival of the wave, instead of numerical oscillations only. Figure 11 shows the difference between a Galerkin formulation of the rezoning equation, where numerical node-to-node oscillations are clear, and a Petrov-Galerkin integration of the free surface equation (i.e. the previous $41 \times 3$ element solution). The temporal criterion [24] is selected for the perturbation of the weighting functions, and, as expected [23,24], better results are obtained if the Courant number is equal to one. In the inviscid dam-break problem over a still fluid, the second-order accurate Newmark scheme [21] is used (i.e. $\gamma=0.5$ and $\beta=0.25$ ), while in all of the following cases numerical damping is necessary (i.e. $\gamma>0.5$ ) because of the small values of $\Delta H$; this numerical instability is discussed later.

For the viscous analysis a reference case is defined using values similar to those used by Jeyapalan [26]. The height of the dam is set equal to $H=15 \mathrm{~m}$; the slope is $2: 1$; the density is $\rho=1600 \mathrm{~kg} / \mathrm{m}^{3}$; and since the viscosity varies between $1 \times 10^{2} \mathrm{~Pa} \cdot \mathrm{s}$ and $1 \times 10^{4} \mathrm{~Pa} \cdot \mathrm{s}$, an average value of $1 \times 10^{3}$ is defined; given these values, the reference Reynolds number is set equal to 300 ; finally, the depth of the downstream still fluid of the dam is taken as $25 \%$ of the total depth of the upstream reservoir because the flow over a dry bed will be studied later.

The influence of the Reynolds number is presented in Fig. 12. It is important to notice the influence of the viscous energy dissipation on the wave velocity (slower than in the inviscid case) and on the oscillations of the free surface. Notice the smoothness of the free surface 
A. Huerta, W.K. Liu, Viscous flow with large free surface motion
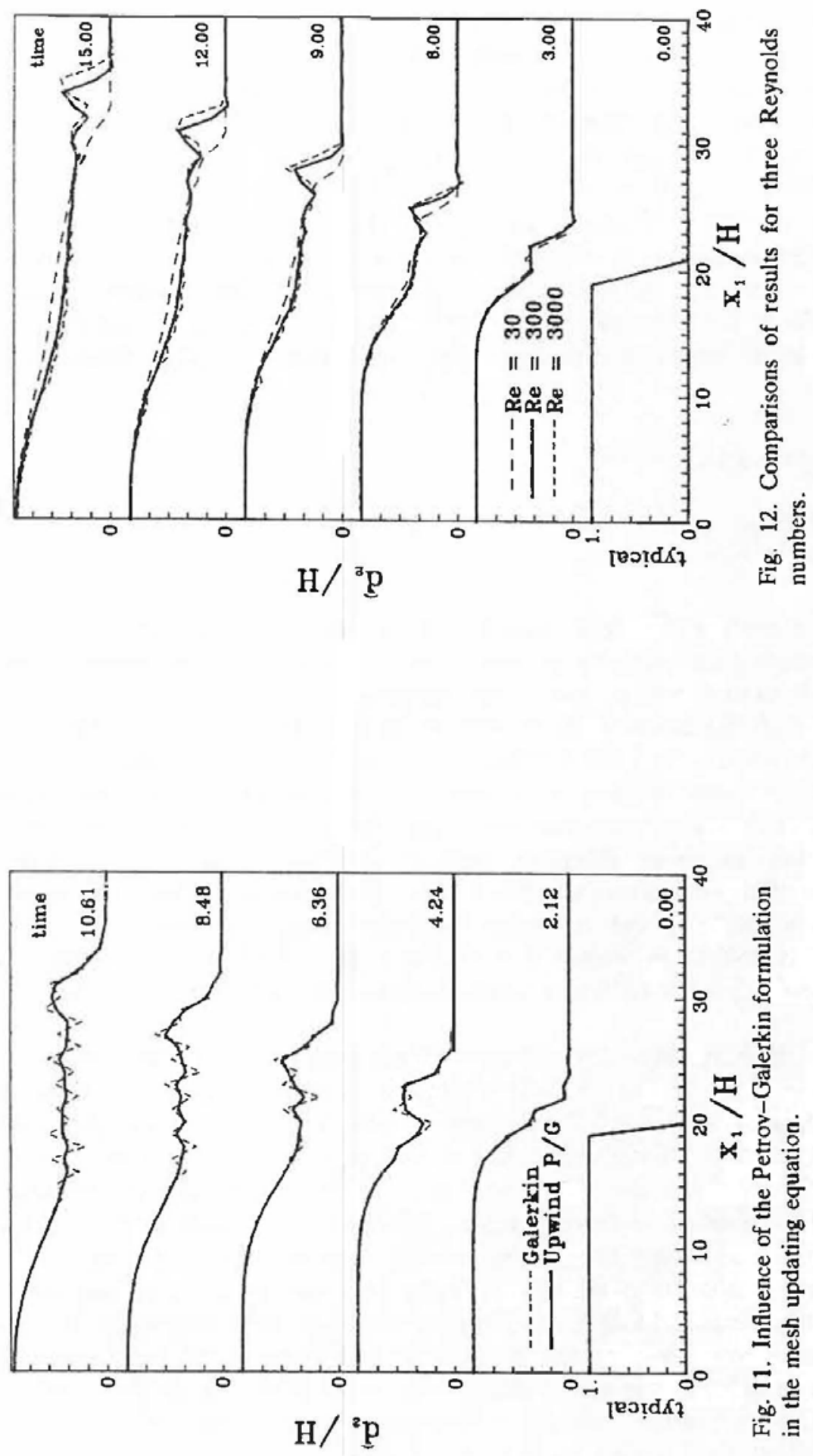


\section{A. Hucria, W.K. Liu, Viscous flow with large free surface motion}

generated by a Reynolds number of 30 and the clear influence in this case of the viscous energy dissipation, while at $\mathrm{Re}=3000$, which is an extremely high value, the free surface displacements are more similar to those of the reference case or even the inviscid case, because all of the shearing is reduced to a small boundary layer. For the highest value of the Reynolds number, seven elements in depth were necessary to capture the boundary layer.

Next, several non-Newtonian cases are studied. In order to avoid comparisons with a lot of material parameters, simplifications of the Carreau and Herschel Bulkley models (see Table 1) are employed, i.e. the Carreau- $\mathrm{A}$ and the Bingham models, respectively. The latter model is, in fact, transformed into a biviscous material with an extremely high initial viscosity because of the numerical impossibility to prescribe infinite viscosities; this is a classic technique employed, for example, by O'Donovan and Tanner [41] and Keentok et al. [28]. The one-dimensional viscosity equations for the Carreau-A and Bingham materials are, respectively,

and

$$
\mu=\mu_{0}\left[1+(\lambda \dot{\gamma})^{2}\right]^{(n-1) / 2}
$$

$$
\mu= \begin{cases}1000 \mu_{\mathrm{p}} & \text { if } \tau \leqslant \tau_{0}, \\ \mu_{\mathrm{p}}+\tau_{0} / \dot{\gamma} & \text { if } \tau \geqslant \tau_{0}\end{cases}
$$

where $\mu, \dot{\gamma}$, and $\tau$ are the absolute viscosity, shear rate, and shear stress, respectively; $\mu_{0}, \lambda$, and $n$ are zero-shear-rate viscosity, a time constant, and the dimensionless power-law index for the Carreau-A model; and $\mu_{\mathrm{p}}$ and $\tau_{0}$ are the plastic viscosity and the plastic yield stress for the Bingham model. Equation (50) is plotted in Fig. 13 for $\mu_{0}=1 \times 10^{4} \mathrm{~Pa} \cdot \mathrm{s}$ (the lowest viscosity studied), $\lambda=5 \mathrm{~s}$, and three values of $n$. At $n=1.0$ the behavior is Newtonian, and for decreasing $n$ the shear thinning is increased. Because no fluid has been found that would exhibit more shear thinning than that corresponding to $n=0.2$ [47], this value is chosen to study the influence of shear thinning, and an average value, i.e. $n=0.6$, between the Newtonian case and the extreme case of $n=0.2$ is also analyzed. Figure 13 also shows the two Bingham models studied; in both cases the yield stress is prescribed to be $1 \times 10^{3} \mathrm{~Pa}$, while the plastic viscosity is taken as either $1 \times 10^{2} \mathrm{~Pa} \cdot \mathrm{s}$ or $1 \times 10^{3} \mathrm{~Pa} \cdot \mathrm{s}$; in fact, the values of $\tau_{0}=1 \times 10^{3} \mathrm{~Pa}$ and $\mu_{\mathrm{p}}=1 \times 10^{3} \mathrm{~Pa} \cdot \mathrm{s}$ were used in the reference case chosen by Jeyapalan [26].

The computed free surfaces for different times and the previous generalized Newtonian fluids are shown in Figs. 14 and 15. It is important to point out that the results obtained with the Carreau-A model and $n=0.2$ are very similar to those of the Newtonian case with $\mathrm{Rc}=300$, whcrcas for the Bingham material with $\mu_{\mathrm{p}}=1 \times 10^{2} \mathrm{~Pa} \cdot \mathrm{s}$ the free surface shapes resemble more closely those associated with $\mathrm{Re}=3000$; this is expected because the range of shear rate for this problem is from 0 up to $2030 \mathrm{~s}^{-1}$. It should also be noticed that both Bingham cases present larger oscillations at the free surface and that even for the $\mu_{\mathrm{\rho}}=$ $1 \times 10^{3} \mathrm{~Pa} \cdot \mathrm{s}$ case the flooding wave moves faster than that for the Carreau models. Two main reasons can explain such behavior; first, unless uneconomical time-steps are chosen, oscillations appear in the areas where the fluid is at rest because of the extremely high initial viscosity $\left(1000 \mu_{\mathrm{p}}\right)$; second, the larger shear rates occur at the tip of the wave, and it in in area that the viscosity suddenly drops at least two orders of magnitude, creating numerical oscillations. This behavior is also present in the flow over a dry bed (FDB) problem. 
A. Huerta, W. K. Liu, Viscous flow with large fre? surface motion
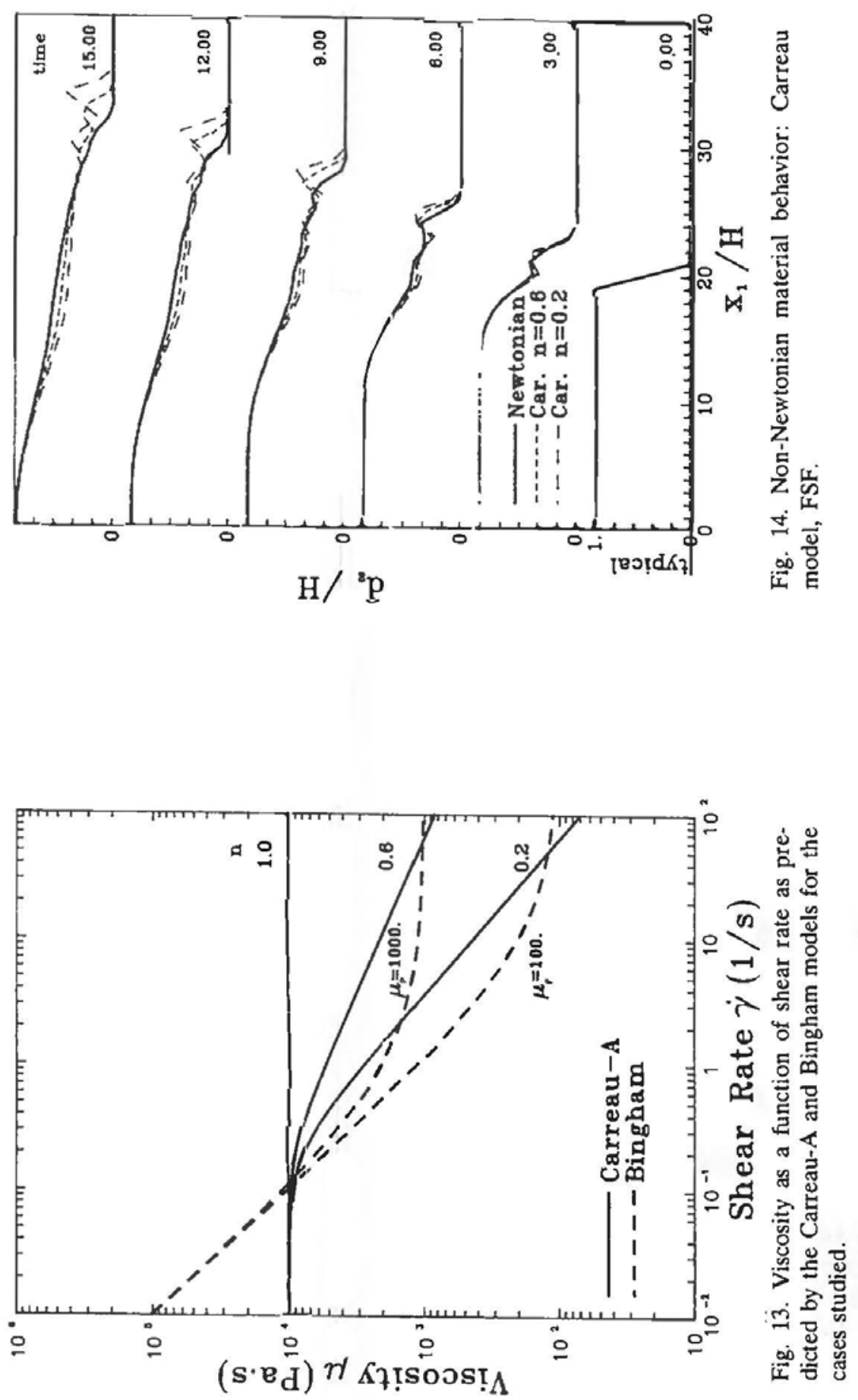
A. Huerta, W.K. Lil, Viscous flow with large free surface motion

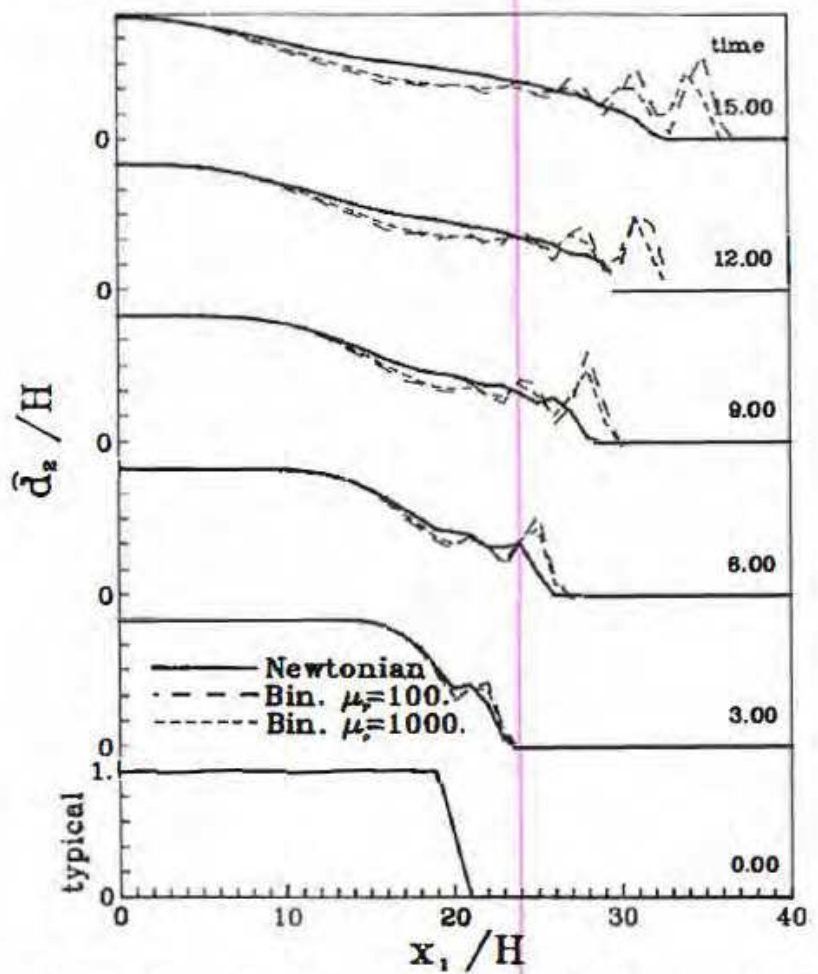

Fig. 15. Non-Newtonian material behavior: Bingham model, FSF.

\subsubsection{Flow over a dry bed}

In the FDB analysis only the downstream boundary condition differs from the FSF problem. This boundary is idealized as a vertical cut of variable depth with a constant horizontal material velocity through depth; for the viscous flows at the bottom of this boundary a zero velocity is prescribed. The physical interpretation for this downstream boundary condition is that the flow is pushing a frictionless vertical wall that does not have any mass or create any opposition to the fluid flow.

Figure 16 shows the finite element mesh (one element in depth) at different instants for the inviscid problem; note the vertical cut of the tip at $t=2 \mathrm{~s}$, it is also important to state that the elements are translated in the horizontal direction. Therefore, for this analysis only the mixed formulation for the mesh rezoning is valid. A Lagrangian description is used for the vertical motion of the free surface and for the horizontal motion of the wave tip. The rest of the nodes are translated by prescribing a horizontal mesh velocity that varies linearly between zero at the upstream boundary and the tip velocity at the downstream boundary. At the tip the vertical mesh velocity $\left(\hat{v}_{2}\right)$ varies linearly with height; for the upstream nodes the same $\hat{v}_{2}$ may be used in order to keep all the interior elements rectangular and to concentrate the number of nodes on the boundary layer, or a linear horizontal reduction of the vertical mesh velocity can be implemented to avoid very small elements at the upstream boundary (see Fig. 20).

The shallow-water theory predicts a parabolic shape for the free surface, a tip velocity of $2 \sqrt{g H}$, and a linear velocity variation between the back of the wave and its leading edge. 

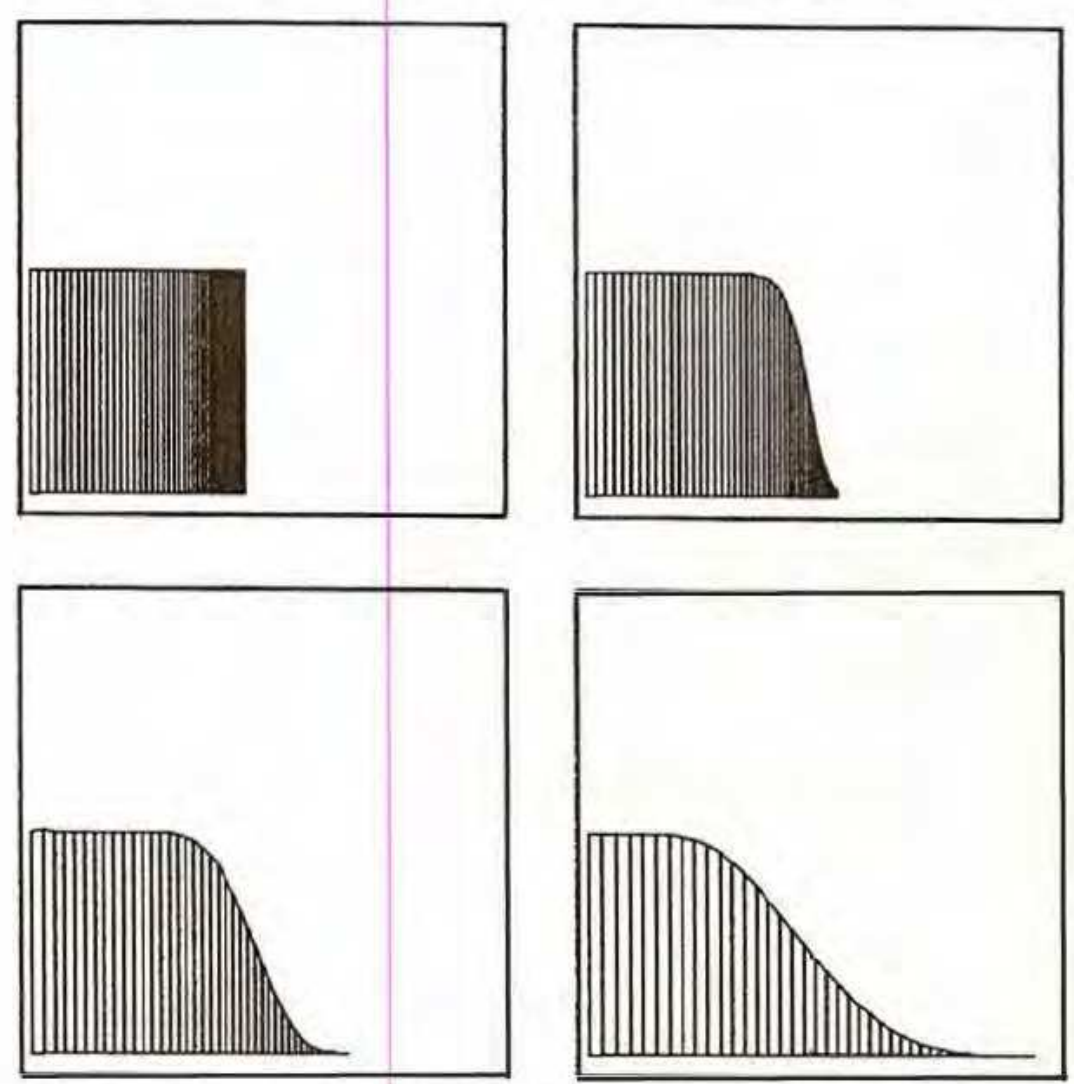

Fig. 16. Finite element mesh motion for the inviscid FDB problem, (a) $t=0$, (b) $t=2$, (c) $t=5$, (d) $t=8$.

Figure 17 shows that the computed free surfaces are close to a parabolic shape and, after two seconds, they all cross at the height predicted by Ritter's solution. However, a small translation of this crossing point is observed and the velocity of the flooding wave tends to $3 \sqrt{g H}$, instead of the expected $2 \sqrt{g H}$ value. The translation and unmatching tip velocity are explained by the fact that the tip acceleration is unrealistic at $t=0^{+}$. After $t=1.5 \mathrm{~s}$, the solution stabilizes and approaches a steady state. Recall that shallow water theory predicts an infinite acceleration at $t=0$ and zero elsewhere. Obviously, this initial acceleration is the cause of the volume change and the final tip velocity; moreover, these oscillations are time-step and element size dependent. In any case, if Ritter's solution at $t=\varepsilon \mathrm{S}$ (small $\varepsilon$ ) is prescribed as initial condition, no volume changes are observed and the tip velocity remains constant at $2 \sqrt{\mathrm{gH}}$.

For the viscous analysis of the FDB problem, the generalized Newtonian fluids dehined before (see Fig. 13) are studied. Figure 18 shows the motion for the three Carreau models, and Fig. 19 compares the surface shapes for $n=0.2$ and the FSF and FDB problems, while Fig. 20 presents the mesh motion for the same fluid. As expected, in the FDB problem, the energy dissipation is considerably larger than in the FSF case, and therefore the wave celerity is decreased. Notice also that the free surface shows less oscillation because the mass transport in the FDB problem is fundamentally horizontal, while in the FSF problem the vertical mass 
A. Huerta, W, K, Liu, Viscous flow with large free surface motion

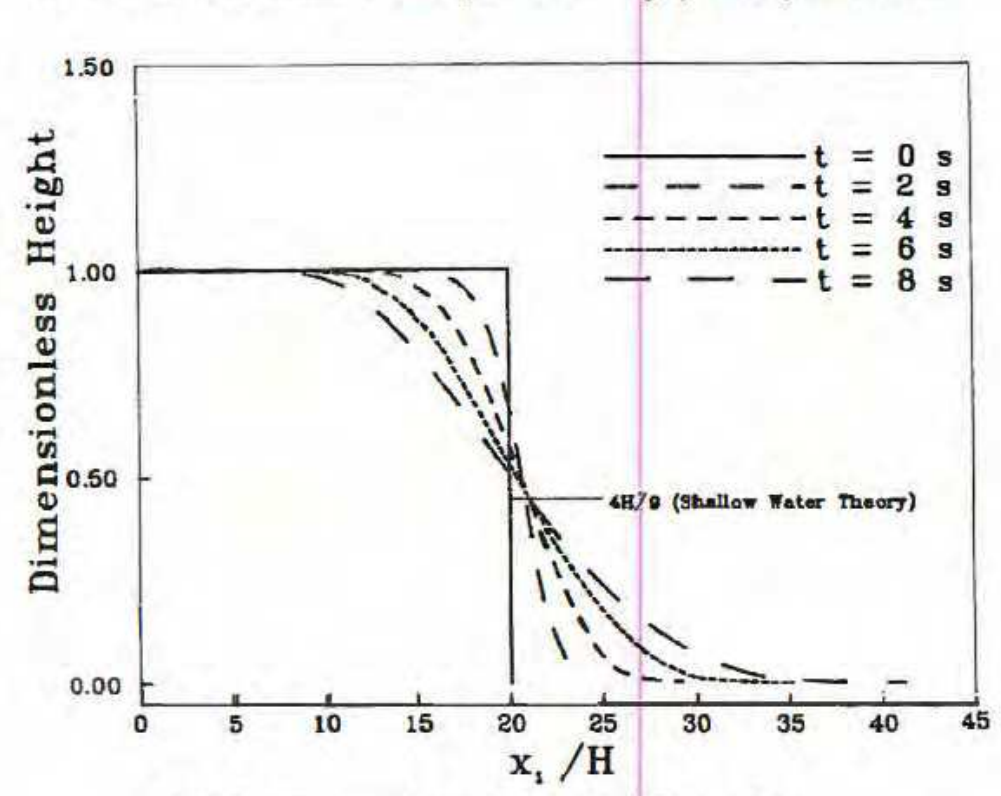

Fig. 17. Free surface results at different times.

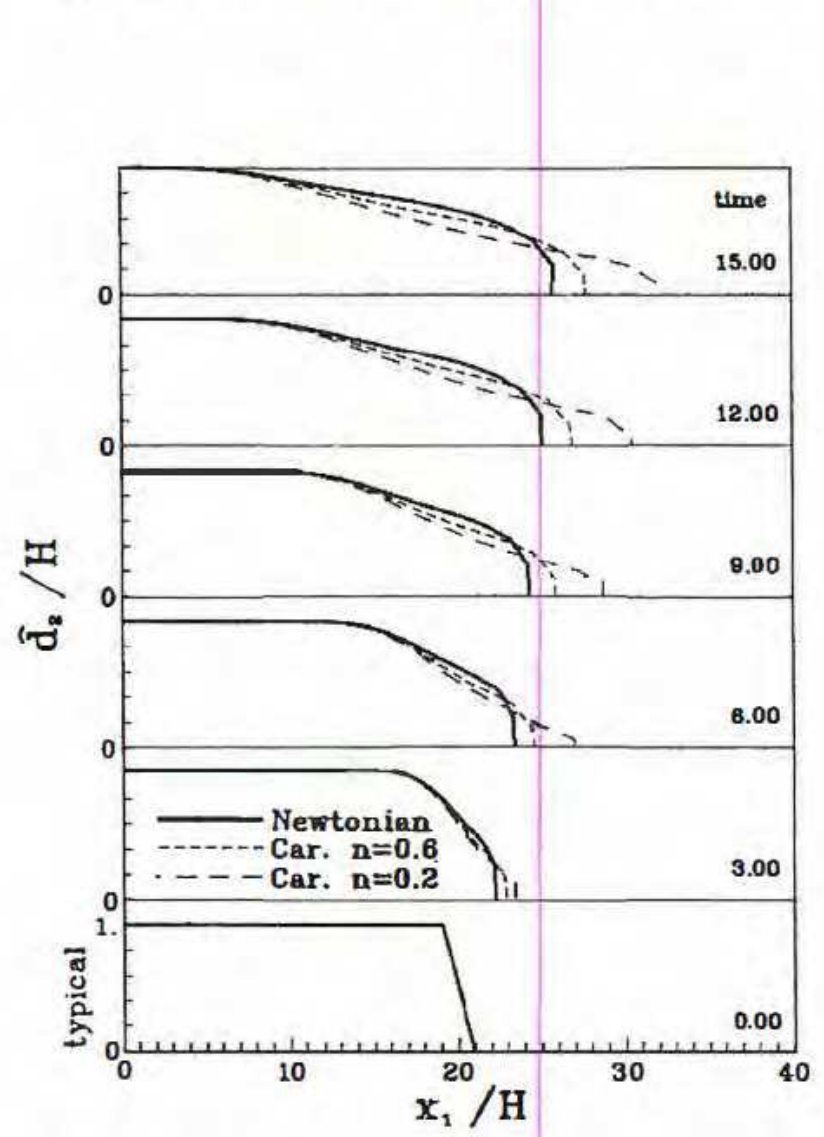

Fig. 18. Non-Newtonian material behavior: Carreau model, FDB. 

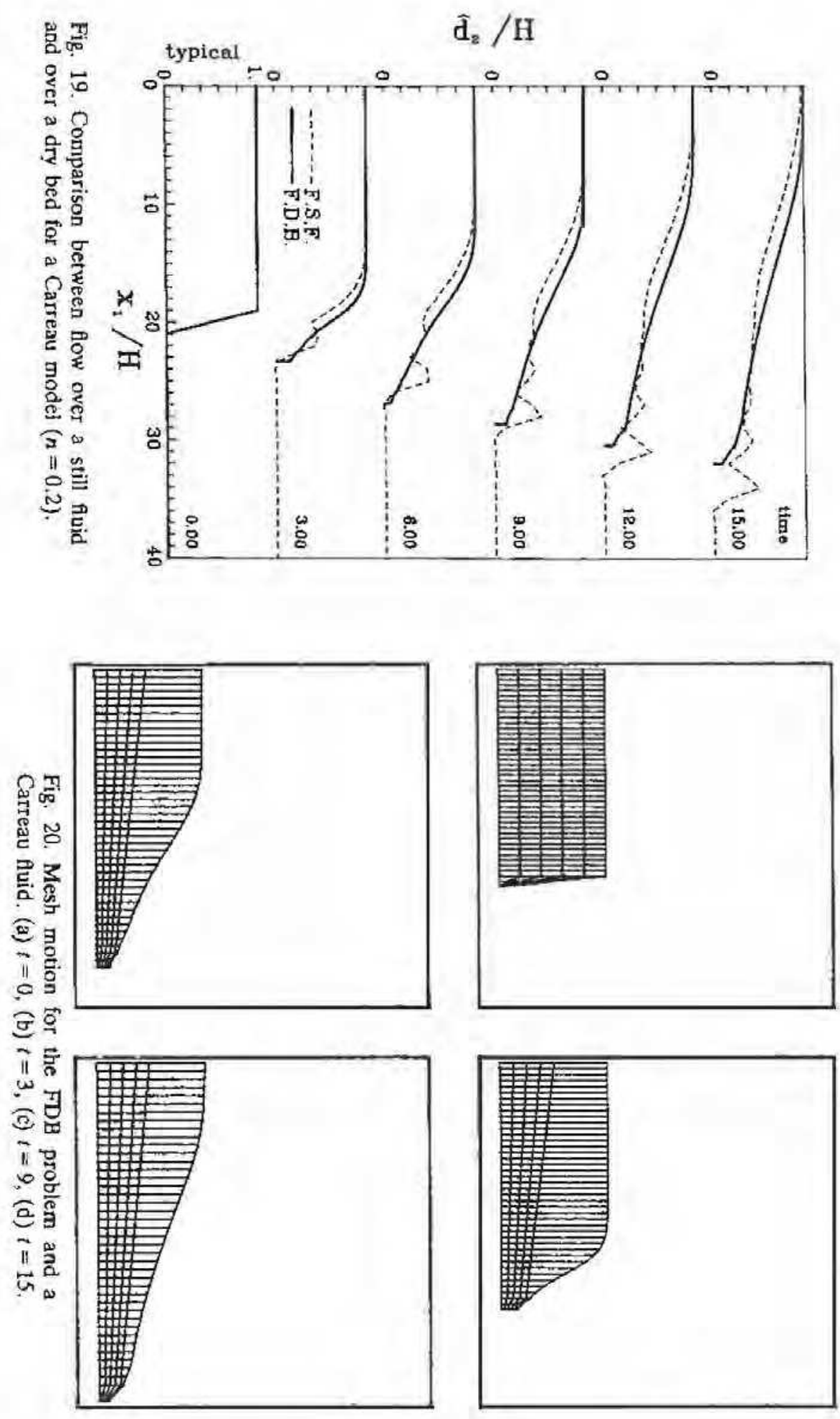

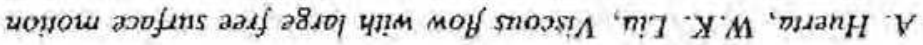




\section{A. Huerta. W.K. Liu, Viscous flow with large free surface motion}

motion is much more important. For the Bingham models only the reference case studied by Jeyapalan [26] (i.e. $\tau_{0}=1 \times 10^{3} \mathrm{~Pa}$ and $\mu_{\mathrm{p}}=1 \times 10^{3} \mathrm{~Pa} \cdot \mathrm{s}$ ) gives a stable solution, whereas for $\mu_{\mathrm{p}}=1 \times 10^{2} \mathrm{~Pa} \cdot \mathrm{s}$ numerical instabilities of the tip velocity create element entanglement. These instabilities, which are damped at low to medium Reynolds numbers, are caused by the particular choice of the downstream boundary condition. Along the vertical tip the horizontal velocities are prescribed equal; this introduces an unrealistic velocity distribution which is different from the 'natural' velocity distribution existent at the nodes just upstream of the tip; hence, the horizontal tip velocity begins to oscillate because it tries to match the preceding natural velocity distribution and consequently induces a sharp oscillation of the free surface at the last element (see Fig, 21). Figure 22 shows the tip displacement varying with time for all of the non-Newtonian cases studied.

Finally, the experimental results conducted by Jeyapalan [26] are predicted by the use of this model. He performed several flume tests wherein a viscous oil $(\mu=3.9 \mathrm{~Pa} \cdot \mathrm{s}$ and $\rho=897.05 \mathrm{~kg} / \mathrm{m}^{3}$ ) was released from an impoundment, $0.1524 \mathrm{~m}$ deep, and with a reservoir length of $4 \mathrm{H}$. The predictions, employing three finite element meshes, are shown in Fig. 23. The over-predicted displacements are probably due to two main reasons; first, the idealization of the upstream vertical wall as frictionless is not accurate for viscous materials, and second, the initial oscillations due to extremely high initial accelerations produce variations in the maximum tip velocity (see Fig. 23(b)) and hence a rotation (i.e. change in slope) of the

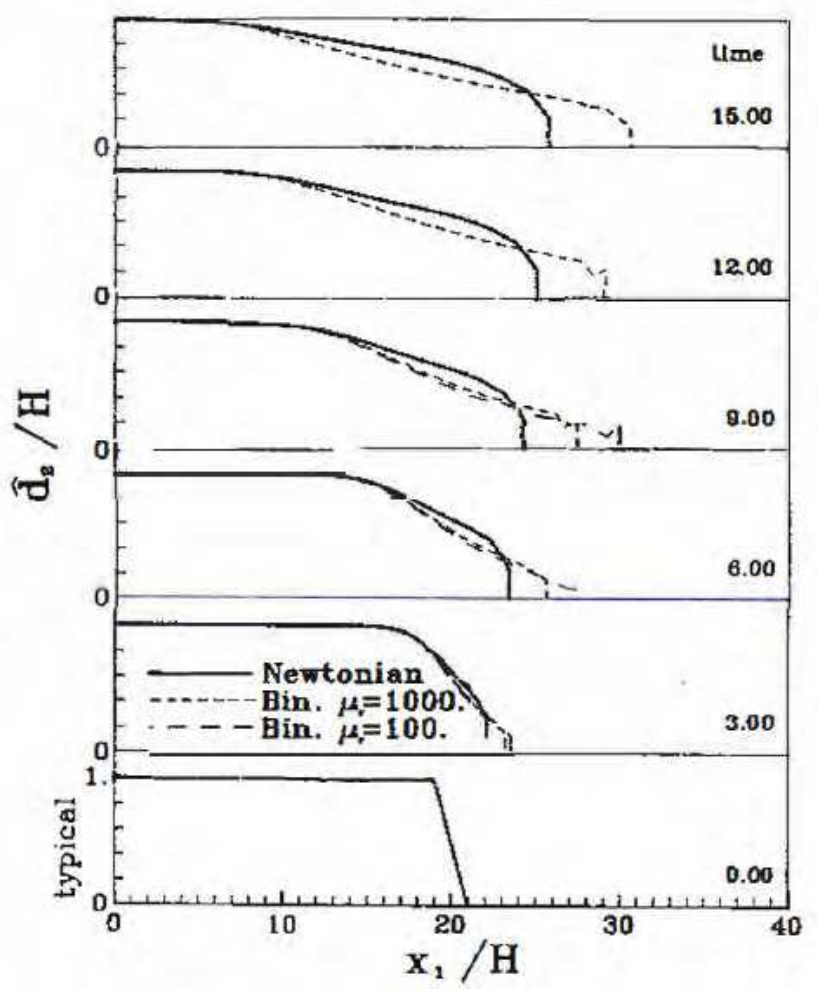

Fig. 21. Non-Newtonian material behavior: Bingham model, FDB. 


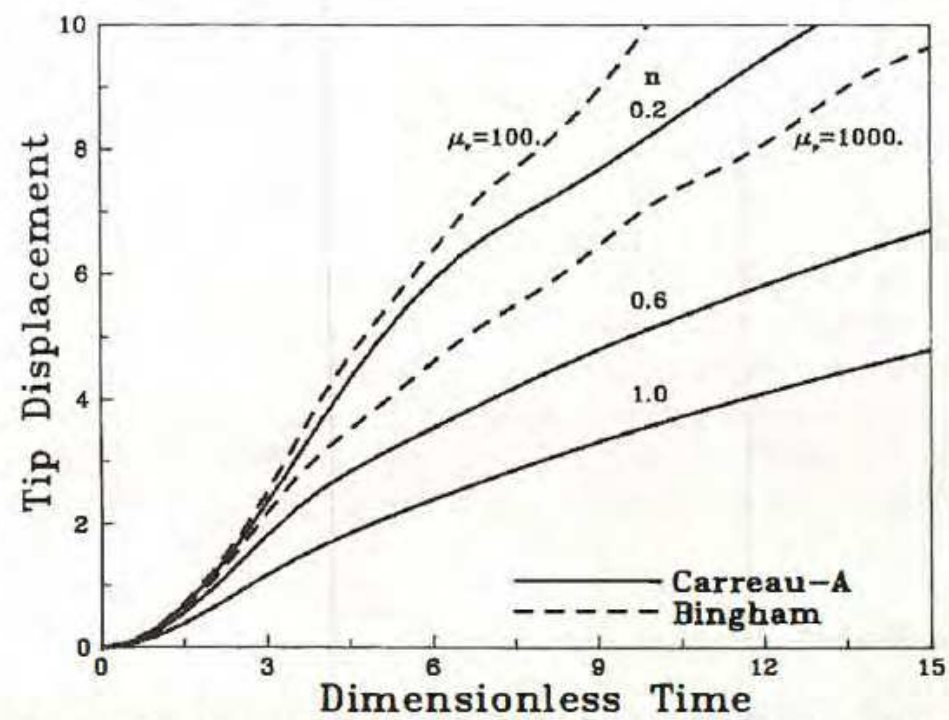

Fig. 22. Tip displacement for the non-Newtonian materials studied.
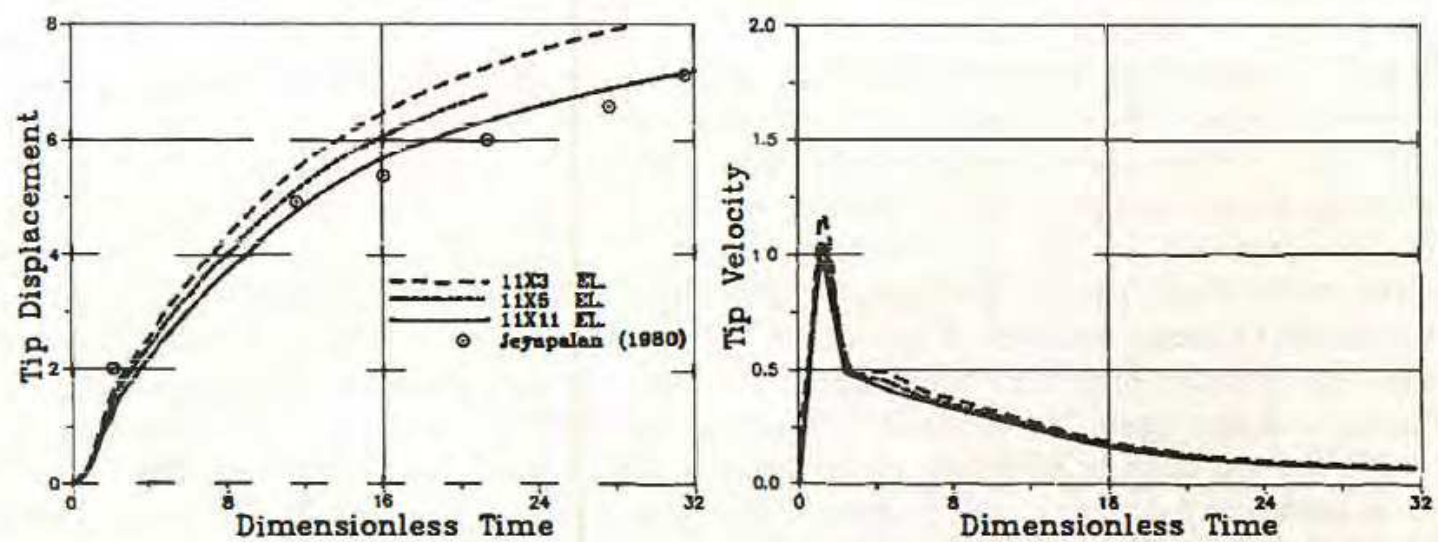

Fig. 23. Comparison of results with experimental data of Jeyapalan [26]. (a) Tip displacement. (b) Tip velocity.

displacement curve near the origin. When the number of elements through the depth is increased, the initial oscillations are decreased and therefore a better prediction is observed.

It is obvious that the ALE finite element analysis of the dam-break problem offers room for improvement, even though an important step forward has been achieved by the complete integration of the field equations through depth and the improved automatic rezoning techniques. Considerable work is being focused on the convection-dominated equations by outstanding researchers; in the case of the dam-break problem better descriptions of the initial instants of flow and the downstream boundary condition are also needed. 


\section{A. Huerto, W.K. Liu, Viscous flow with large free surface motion}

\subsection{Large-amplitude sloshing}

The complexity of seismic huid-structure interaction problems is often accentuated by the free surface motion, in particular when studying the sloshing response of liquid-filled tanks, fuel storage pools, and nuclear reactor systems. The sloshing behavior is determined with a rigid-wall assumption when an uncoupled approach between fluid and structure is chosen $[3,33,37,49]$, or when overflow of liquid during an earthquake in fuel storage pools is studied [39]. Lagrangian updating techniques with some modifications to avoid excessive mesh distortions [42] have been implemented but either the time-step constraints are too severe, or the wave amplitudes are small (approximately 1\% of the depth). Here, the ALE description is used to study large-amplitude sloshing (in the order of $50 \%$ of the depth).

The two-dimensional rigid container has a depth $D$ and a width $W$, it is excited by an acceleration

$$
g_{1}=A g \sin (\omega t)
$$

where $\xi, \omega$, and $t$ are the acceleration of gravity, circular frequency, and time, respectively, and $A$ is an arbitrary constant governing the amplitude of the excitation. After scaling the problem using $D$ as the characteristic length, $\sqrt{g} D$ as the velocity scale, and $\rho g D$ as the characteristic pressure, the governing dimensionless parameters are

$$
\alpha=D / W, \quad A, \quad \tilde{\omega}=\omega \sqrt{D / g} \text { and } \operatorname{Re}=D \sqrt{g} D / \nu
$$

where $\alpha$ is associated with the geometry, $A$ and $\tilde{\omega}$ are the parameters defining the excitation, $\mathrm{Re}$ is the classical Reynolds number, and $\nu$ is the kinematic viscosity.

In order to compare the numerical results with the experimental tests described in [39] the following values are prescribed; $D=0.3 \mathrm{~m}, W=0.8 \mathrm{~m}, A=0.01(10 \mathrm{Gal})$ for the first mode and 0.03 ( $30 \mathrm{Gal}$ ) for the third mode, and $\mu=10^{-3} \mathrm{~Pa} \cdot \mathrm{s}$ (water). Due to the almost inviscid nature of the fluid, perfect frictionless boundaries are assumed between liquid and tank. The finite element mesh consists of $441(21 \times 21)$ constant pressure elements, thus 484 nodes are used. The Petrov-Galerkin formulation is used for the equilibrium equation. On the free surface, a Lagrangian description is used in the vertical direction, and the vertical mesh velocity for the interior elements varies linearly with depth. An Eulerian description is chosen in the horizontal direction everywhere. The time step for the first sloshing mode analysis is taken equal to $\pi / 30$, i.e. 60 time steps per cycle if the impressed frequency is $0.91 \mathrm{~Hz}$.

After ten cycles the maximum wave height is measured and Fig. 24 shows its variation with frequency. The resonance frequency obtained $(0.89 \pm 0.01 \mathrm{~Hz})$ compares well with the ex perimental tests $(0.88 \mathrm{~Hz})$, finite element analysis $(0.898 \mathrm{~Hz})$, and Houssner's theory $(0.902 \mathrm{~Hz})$, results reported in [39]. Figure 25 presents two instantaneous configurations of the domain and the streamlines; it is interesting to notice the vertical motion of the free surface at the center of the tank where the vertical material velocity is always equal to zero. The same phenomena is observed in Fig. 26 where the free surface is plotted at 12 different instants for one cycle, Muto et al. [39] show similar figures which are photographs of the liquid motion taken with slow shutter speeds. These nonlinearities which are caused by the convective term in the mesh updating equation, are also clear in Fig. 27, where the vertical motion of the wave at the tank wall is plotted. Notice that both nodes at opposite sides of the tank have a phase 
A. Huertu, W.K. Liu, Viscous flow with large free surface motion

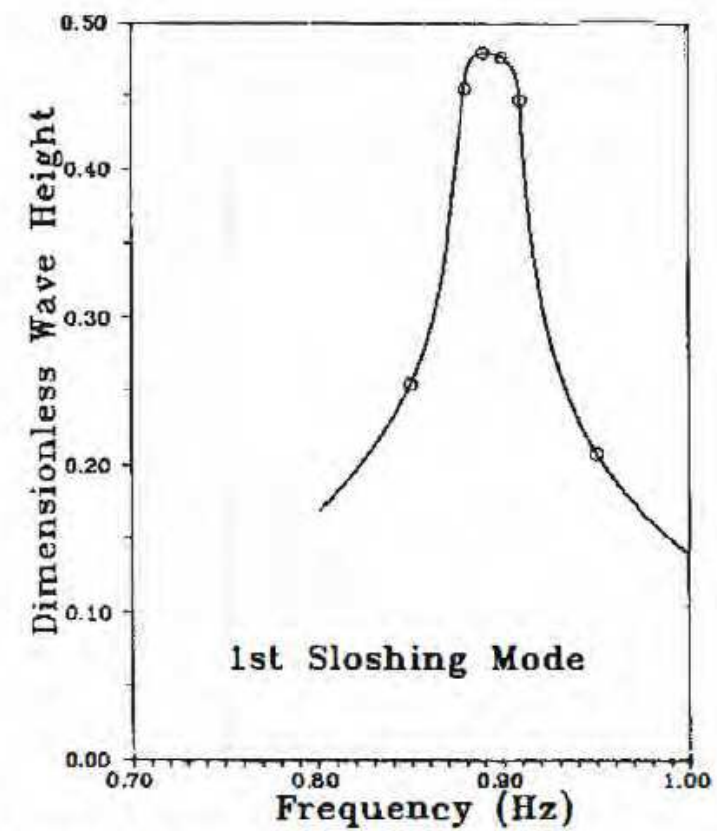

Fig. 24. Upward sloshing wave height at left end versus frequency.
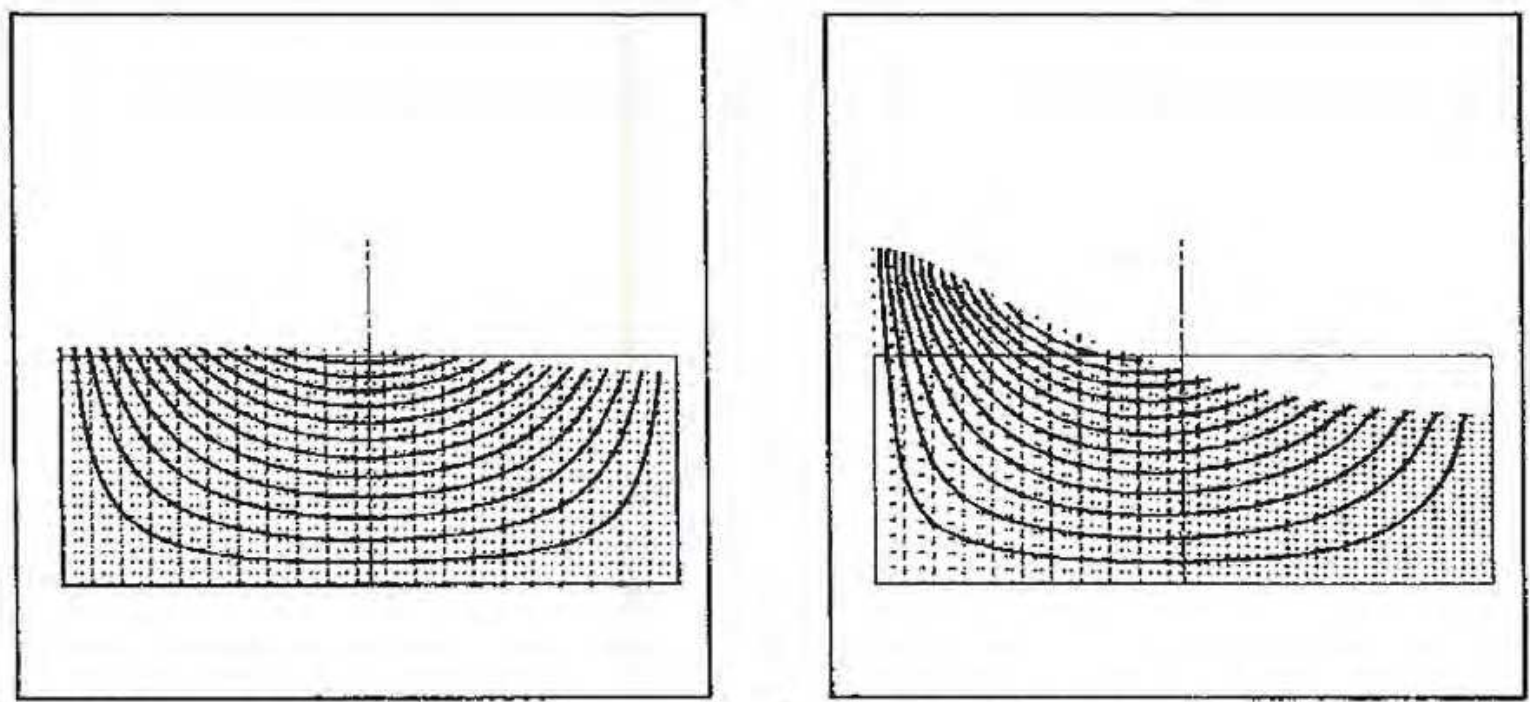

Fig. 25. Instantaneous domain configurations and streamlines for the first sloshing mode at (a) $t=n 2 \pi-\pi / 2$, and (b) $\omega t=n 2 \pi$.

lag of half a cycle, and that the upward displacement is twice the downward motion. The latter figure corresponds to a forced vibration at the resonance frequency during ten cycles before free oscillations occur. Almost no damping is observed because the viscosity of water is very small, the Petrov-Galerkin formulation does not overdamp the solution, and frictionless walls are assumed. 
A. Huerta, W. K. Liu, Viscous flow with large free surface motion

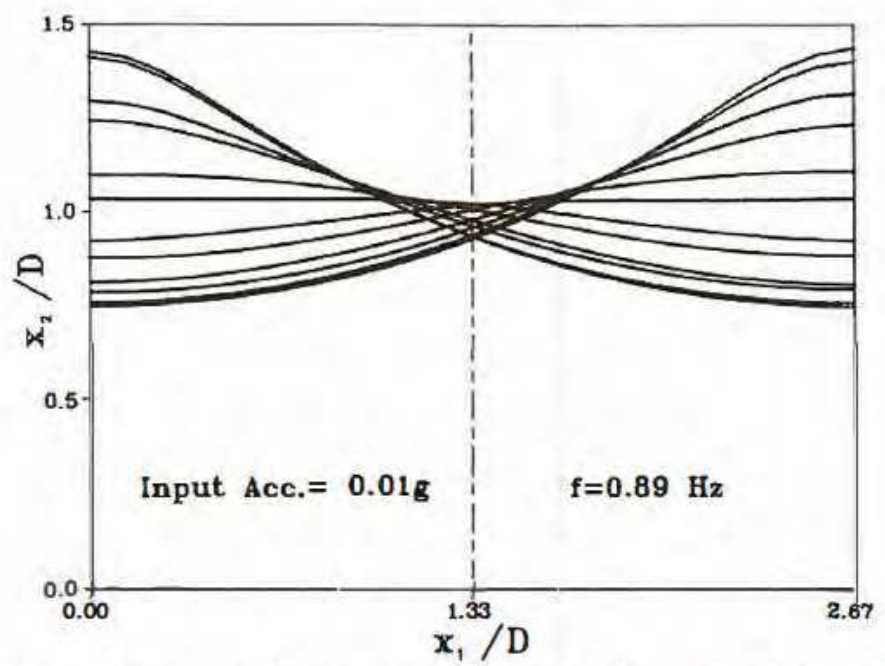

Fig. 26. Instantaneous free surfaces for one cycle-first sloshiug mode.

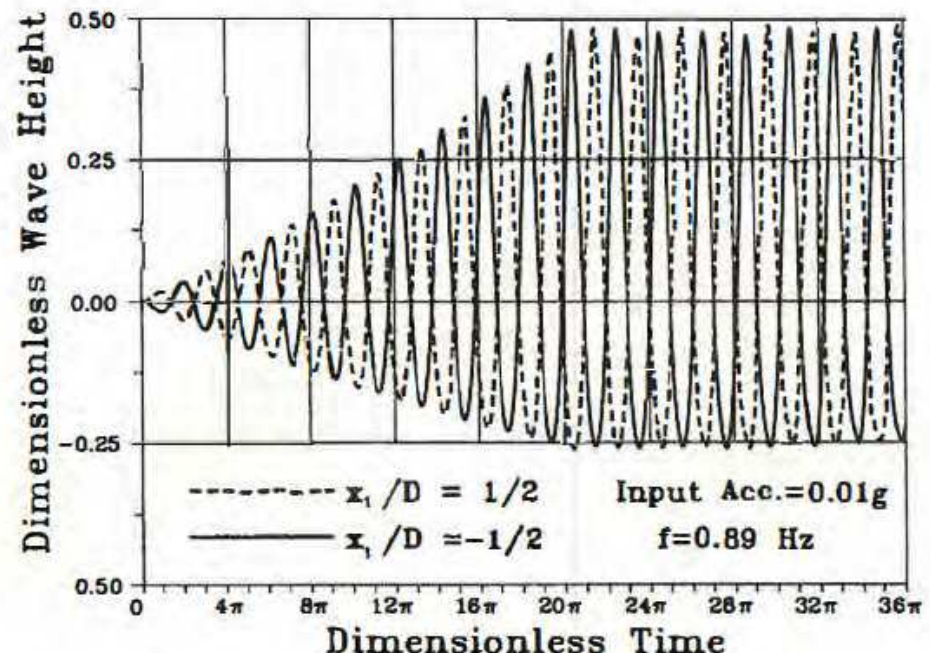

Fig. 27. Time histories of the wave heights at the wall.

Finally, the third sloshing mode is simulated by imposing the empirical resonance frequency $(1.67 \mathrm{~Hz})$ found in [39]. Sixty time steps are also taken for each of the 15 cycles with an input acceleration three times larger than for the first sloshing mode. Figure 28 shows two instantaneous configurations, notice that at $\omega t=n 2 \pi$ ( $n$ being an integer) the free surface amplitude is again maximum and vortices are clearly observed, while at $\omega t=n 2 \pi-\pi / 2$ a symmetric streamline pattern associated with maximum velocity amplitudes predetermines the free surface displacements. This latter figure agrees perfectly with the conclusion of Muto et al. [39] that the third mode has small water movement in the lower half of the container. 

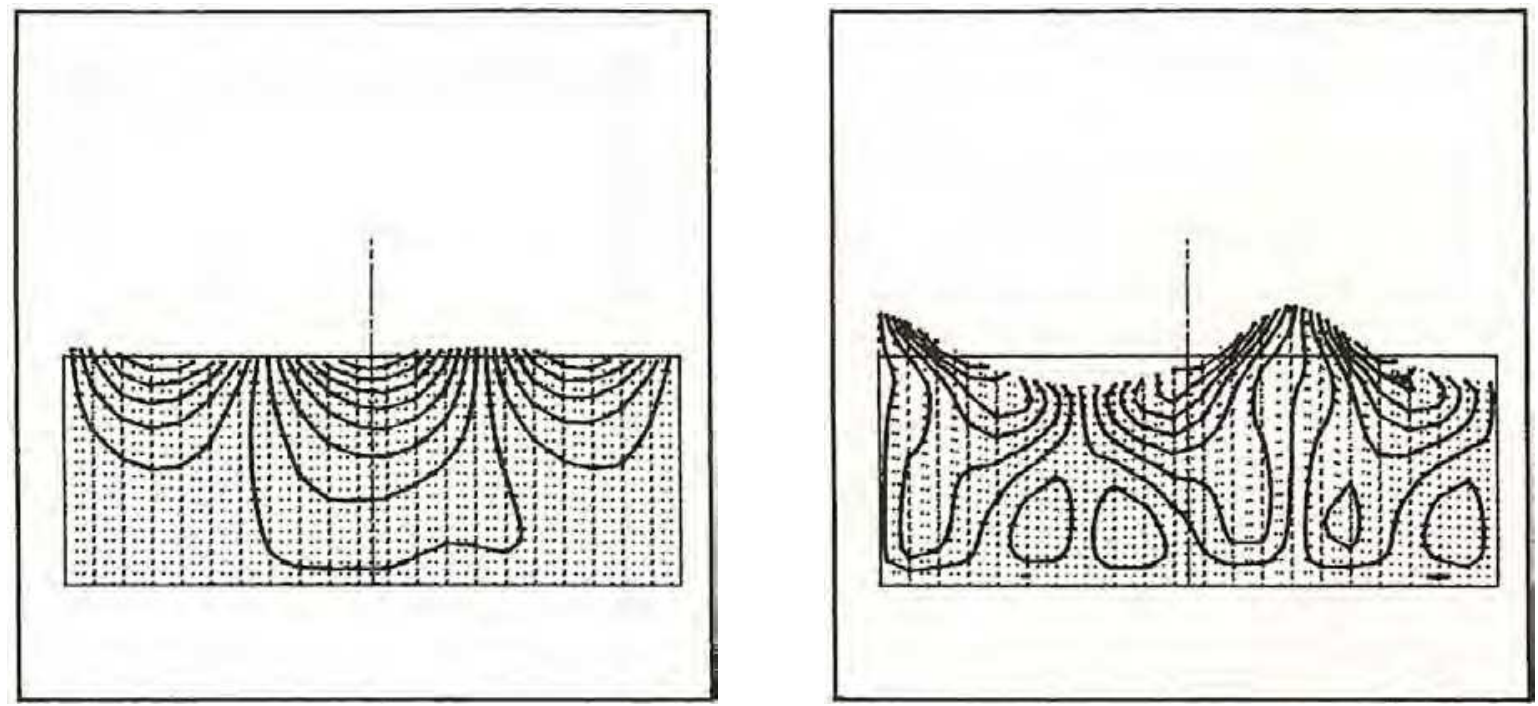

Fig. 28. Instantaneous domain configurations and streamlines for the third sloshing mode at (a) $t=n 2 \pi-\pi / 2$, and (b) $\omega t=n 2 \pi$.

Figure 29 presents 12 instantaneous free surfaces at the fifteenth cycle, and again, the nonlinear treatment of the free surface accounts for the vertical motion of the points that have zero vertical material velocity. The good agreement obtained between the experimental results and the numerical model indicates that the ALE technique presented here may be used for fluid-structure interaction analysis with large boundary motion.

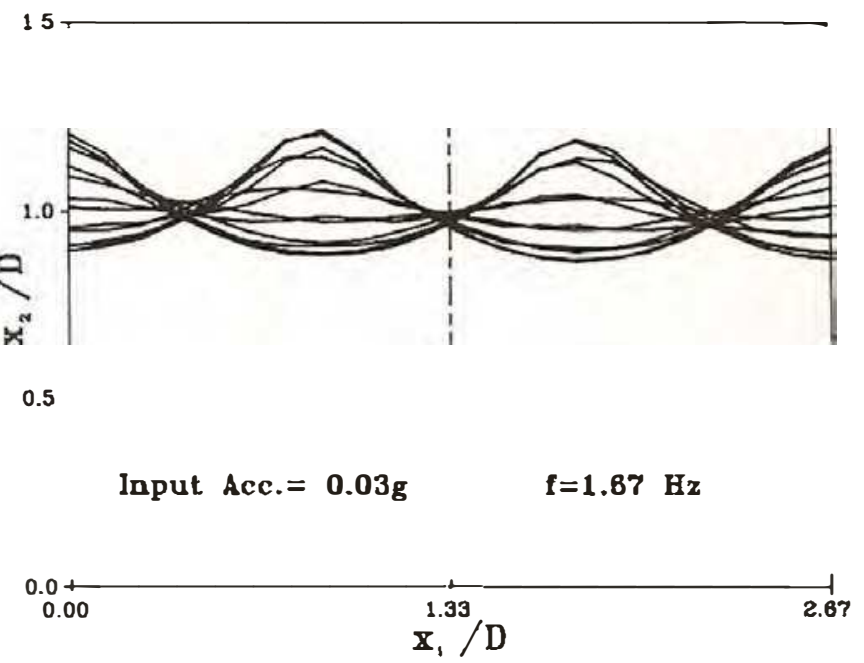

Fig. 29. Instantaneous free surfaces for one cycle-third sloshing mode. 


\section{Conclusions}

This paper illustrates the application of the arbitrary Lagrangian-Eulerian techniques to free surface viscous flows. Because of the use of a pressure velocity mixed formulation and the improvements in accuracy of the streamline upwind Petrov-Galerkin techniques, the computer program is implemented on an IBM AT pcrsonal computer in single precision. Moreover, various updating methods are developed, including a new mixed formulation which is able to map the moving domain in a more rational manner. A special feature of this method is the use of a streamline-upwind/Petrov-Galerkin formulation for the mesh updating equation. Clear progress has been ohtained in the numerical modeling of the tsunami, large amplitude sloshing, and dam break problems. The latter also incorporates the non Newtonian behavior of the material. In conclusion, the ALE method allows an efficient and accurate description of large free surface motions. This is not the case in the pure Eulerian or Lagrangian descriptions.

\section{Appendix A. Conservation of mass and momentum in referential form}

\section{A.1. Conservation of mass (equation of continuity)}

The interest here is to derive the principle of mass conservation in referential form. Consider an arbitrary volume $V_{x}$ fixed in the referential domain, $R_{x}$, and bounded by the surface $\partial V_{x}$; the medium is continuous and with a density $\hat{\rho}(\boldsymbol{x}, t)$. In fact, using classical transformation of coordinates between the volume $V_{x}$ and its spatial, $V_{x}$, and material, $V_{x}$, representations, one can write that at time $t$ the total mass in $V_{x}$ is

where

$$
M=\int_{V_{x}} \hat{\rho} \mathrm{d} \Omega_{X}=\int_{V_{x}} \rho \mathrm{d} \Omega_{x}=\int_{V_{X}} \rho^{0} \mathrm{~d} \Omega_{X},
$$

$$
\begin{aligned}
& \hat{\rho}(\chi, t)=\hat{J} \rho(x, t), \\
& \rho^{0}\left(X, t_{0}\right)=J \rho(x, t), \\
& \hat{J}=\operatorname{det}\left[\frac{\partial x_{i}}{\partial x_{j}}\right], \\
& J=\operatorname{det}\left[\frac{\partial x_{i}}{\partial X_{j}}\right] .
\end{aligned}
$$

The principle of mass conservation states that the local rate of increase of the total mass in the volume

$$
\left.\partial\right|_{x}-\left.\int_{V_{x}}^{\partial t}\right|_{x} \mathrm{~d} \Omega_{x}
$$

must be equal, if no mass is created or destroyed inside $V_{x}$, to the rate of inflow of mass 
through the bounding surface $\partial V_{x}$, namely

$$
-\int_{\partial V_{x}} \hat{\rho} w \cdot \hat{n} \mathrm{~d} S_{\chi} .
$$

Hence,

$$
\left.\int_{V_{x}} \frac{\partial \hat{\rho}}{\partial t}\right|_{x} \mathrm{~d} \Omega_{\chi}+\int_{\partial V_{x}} \hat{\rho} w \cdot \hat{n} \mathrm{~d} S_{x}=0
$$

where $\hat{\boldsymbol{n}}$ is the outward unit normal to $\partial V_{\chi}$.

Equation (A.4) can be deduced directly from (A.1) using the following procedure. The total rate of change (i.e. material time derivative) of the mass contained in the material volume $V_{X}$ at $t_{0}$, which occupies at time $t$ the referential volume $V_{x}$, must be zero. Thus, from (A.1),

$$
\left.\frac{\partial M}{\partial t}\right|_{X}=\left.\frac{\partial}{\partial t}\right|_{X} \int_{V_{X}} \rho^{0} \mathrm{~d} \Omega_{X}=\left.\frac{\partial}{\partial t}\right|_{X} \int_{V_{X}} \hat{\rho} \mathrm{d} \Omega_{X}=0,
$$

and using the Reynolds transport theorem in referential form (see (19) in Section 2.3) equation (A.4) is obtained:

$$
\left.\begin{gathered}
\partial M \\
\partial t
\end{gathered}\right|_{x}=\left.\int_{V_{x}} \partial \hat{\rho}\right|_{x} \mathrm{~d} \Omega_{x}+\int_{\partial V_{x}} \hat{\rho} w \cdot \hat{n} \mathrm{~d} S_{x}=0 .
$$

Using now the divergence theorem, the previous equation may be rewritten as

$$
\int_{V_{\chi}}\left[\left.\frac{\partial \hat{\rho}}{\partial t}\right|_{\chi}+\frac{\partial \hat{\rho} w_{i}}{\partial \chi_{i}}\right] \mathrm{d} \Omega_{\chi}=0 \text {, }
$$

and since the integral in (A.6) vanishes identically for any arbitrary choice of volume $V_{x}$, the integrand must vanish at every point in the referential domain; hence, the continuity equation in referential form is obtained

$$
\left.\frac{\partial \hat{\rho}}{\partial t}\right|_{\chi}+\frac{\partial \hat{\rho} w_{i}}{\partial \chi_{i}}=0 \text { in } R_{\chi} .
$$

If the Lagrangian description is used, the preceding equation is transformed using

$$
x=X, \quad w=0, \quad \hat{J}=J=\operatorname{det}\left[\frac{\partial x_{i}}{\partial X_{j}}\right], \quad \hat{\rho}=\rho^{0}
$$

to

$$
\left.\begin{gathered}
\partial \rho J \\
\partial t
\end{gathered}\right|_{X}=0 \text { or } \rho J=\rho^{0} \quad \text { in } R_{X} .
$$

On the other hand, if the Eulerian description is taken,

$$
x=x, \quad w=v, \quad \hat{\jmath}=1, \quad \hat{\rho} \quad \rho,
$$


and

$$
\left.\frac{\partial \rho}{\partial \imath}\right|_{x}+\frac{\partial \rho v_{i}}{\partial x_{i}}=0 \text { in } R_{x}
$$

where (A.9) and (A.11) are the two classical forms of the continuity equation, see [38].

Hughes et al. [22] used a different form of the continuity equation which can be obtained directly from (A.1) after differentiating with respect to time holding $\boldsymbol{X}$ fixed,

$$
\left.\frac{\partial M}{\partial t}\right|_{\chi}=\left.\frac{\partial}{\partial t}\right|_{x} \int_{V_{x}} \rho \mathrm{d} \Omega_{x}=0,
$$

using the classical Reynolds transport theorem and the divergence theorem

$$
\int_{V_{x}}\left[\left.\frac{\partial \rho}{\partial t}\right|_{x}+\frac{\partial \rho v_{i}}{\partial x_{i}}\right] \mathrm{d} \Omega_{x}=0
$$

or

$$
\int_{v_{x}}\left[\left.\frac{\partial \rho}{\partial t}\right|_{x}+v_{i} \frac{\partial \rho}{\partial x_{i}}+\rho \frac{\partial v_{i}}{\partial x_{i}}\right] \mathrm{d} \Omega_{x}=0 .
$$

Observing that the first two terms yield the material derivative of $\rho$ and using (13), equation (A.12c) becomes

$$
\int_{V_{x}}\left[\left.\frac{\partial \rho}{\partial t}\right|_{x}+c_{i} \frac{\partial \rho}{\partial x_{i}}+\rho \frac{\partial v_{i}}{\partial x_{i}}\right] \mathrm{d} \Omega_{x}=0,
$$

and since $V_{x}$ is arbitrarily chosen, it follows

$$
\left.\frac{\partial \rho}{\partial t}\right|_{x}+c_{i} \frac{\partial \rho}{\partial x_{i}}+\rho \frac{\partial v_{i}}{\partial x_{i}}=0 \text { in } R_{x} .
$$

\section{A.2. Conservation of momentum (equilibrium equation)}

Using the same definitions as in the conservation of mass, the principle of conservation of momentum states that the total rate of change of the total momentum of the medium occupying at time $t$ the referential volume $V_{x}$,

$$
\left.\frac{\partial}{\partial t}\right|_{x} \int_{v_{x}} \hat{\rho}(x, t) v(x, t) \mathrm{d} \Omega_{x},
$$

is equal to the net force exerted on it,

$$
\int_{\partial V_{x}} \hat{\boldsymbol{t}} \mathrm{d} S_{x}+\int_{V_{x}} \hat{\rho} g \mathrm{~d} \Omega_{x}
$$

where $\hat{t}$ is the force per unit area acting on the surface $\partial V_{x}$ of the volume $V_{x}$, and $g$ is the body force per unit of mass acting in $V_{x}$. The force on the deformed spatial surface per unit of referential area, $\hat{t}$, may be written as a function of the first Piola-Kirchhoff stress tensor $T$ and the outward unit normal, $\hat{n}$, to the referential surface as 


$$
\hat{i}_{i}=\hat{T}_{j i} \hat{n}_{j}
$$

It should be noted that the first Piola-Kirchhoff stress tensor is defined here in the referential sense, i.e. it is defined with respect to the fixed referential domain. Moreover, $\hat{\boldsymbol{T}}$ is related to the Cauchy stress tensor $\sigma$ and to the first Piola Kirchhoff stress tensor in its classical sense $\boldsymbol{T}^{\mathbf{0}}$ (i.e. defined with respect to the material surface at $t_{0}$ ) by the fact that all of them give the same force on the deformed surface, $\mathrm{d} S_{x}$, but use different exterior unit normals and unit surfaces, namely

$$
(\hat{\boldsymbol{n}} \cdot \hat{\boldsymbol{T}}) \mathrm{d} S_{\chi}=(\boldsymbol{n} \cdot \boldsymbol{\sigma}) \mathrm{d} S_{x} \quad\left(\boldsymbol{n}^{0} \cdot T^{0}\right) \mathrm{d} S_{X},
$$

or (see [38])

$$
\begin{aligned}
& T_{i j}^{0}=J \frac{\partial X_{i}}{\partial x_{k}} \sigma_{k j}, \\
& \hat{T}_{i j}=\hat{J} \frac{\partial \chi_{i}}{\partial x_{k}} \sigma_{k j},
\end{aligned}
$$

where $n$ and $n^{0}$ are the exterior unit normals to the deformed surface $\mathrm{d} S_{x}$ and to the material surface at time $t_{0} \mathrm{~d} S_{X}$, respectively.

Substituting (A.15) into (A.14) and using the divergence theorem to transform the surface integral into a volume integral, one gets

$$
\left.\frac{\partial}{\partial t}\right|_{x} \int_{V_{x}} \hat{\rho} v_{i} \mathrm{~d} \Omega_{\chi}=\int_{V_{\chi}}\left[\frac{\partial \hat{T}_{j i}}{\partial \chi_{j}}+\hat{\rho} g_{i}\right] \mathrm{d} \Omega_{\chi} .
$$

The right-hand side in the above equation is transformed using the Reynolds transport theorem and the divergence theorem into

$$
\int_{V_{\chi}}\left[\left.\frac{\partial \hat{\rho} v_{i}}{\partial t}\right|_{\chi}+\begin{array}{c}
\partial w_{j} \hat{\rho} v_{i} \\
\partial \chi_{j}
\end{array}\right] \mathrm{d} \Omega_{\chi}-\int_{V_{\chi}}\left[\frac{\partial \hat{T}_{j i}}{\partial \chi_{j}}+\hat{\rho} g_{i}\right] \mathrm{d} \Omega_{\chi}
$$

which is reduced to

$$
\left.\frac{\partial \hat{\rho} v_{i}}{\partial t}\right|_{x}+\frac{\partial w_{j} \hat{\rho} v_{i}}{\partial \chi_{j}}=\frac{\partial \hat{T}_{j i}}{\partial \chi_{j}}+\hat{\rho} g_{i} \text { in } R_{X},
$$

after noticing that $V_{x}$ is arbitrarily chosen. Equation (A.19) can further be simplified by using the continuity equation, i.e. (A.7); and finally, the equilibrium equation in referential form may be written as

$$
\left.\hat{\rho} \frac{\partial v_{i}}{\partial t}\right|_{x}+\hat{\rho} w_{j} \frac{\partial v_{i}}{\partial \chi_{j}}=\frac{\partial \hat{T}_{j i}}{\partial \chi_{j}}+\hat{\rho} g_{i} \text { in } R_{x} .
$$


The particularizations of the above equation to the Lagrangian and Euler:an descriptions are easily obtained after the corresponding substitutions, i.e. (A.8) and (A.10), are made. In the Lagrangian form one gets

$$
\left.\rho^{0} \frac{\partial v_{i}}{\partial t}\right|_{x}=\frac{\partial T_{j i}^{0}}{\partial X_{j}}+\rho^{0} g_{i} \text { in } R_{X}
$$

because $\hat{\boldsymbol{T}}=\boldsymbol{T}^{0}$. In the Eulerian form

$$
\left.\rho \frac{\partial v_{i}}{\partial t}\right|_{x}+\rho v_{i} \frac{\partial v_{i}}{\partial x_{j}}=\frac{\partial \sigma_{j i}}{\partial x_{j}}+\rho g_{i} \text { in } R_{x},
$$

since $\hat{\boldsymbol{T}}=\boldsymbol{\sigma}$. Equations (A.21) and (A.22) may be found in [38].

The formulation used in [22] is readily obtained once we notice, from (A.16a), that

and that

$$
\int_{V_{x}} \hat{n} \cdot \hat{T} \mathrm{~d} S_{x}=\int_{V_{x}} n \cdot \sigma \mathrm{d} S_{x},
$$

$$
\mathrm{d} \Omega_{x}=\hat{\jmath} \mathrm{d} \Omega_{x} .
$$

Hence, (A.18) is rewritten as

$$
\int_{V_{x}} \hat{J}^{-1}\left[\left.\frac{\partial \hat{\rho} v_{i}}{\partial t}\right|_{x}+\frac{\partial w_{j} \hat{\rho} v_{i}}{\partial \chi_{j}}\right] \mathrm{d} \Omega_{x}=\int_{V_{x}}\left[\frac{\partial \sigma_{j i}}{\partial x_{j}}+\hat{\rho} g_{i} \hat{I}^{-1}\right] \mathrm{d} \Omega_{x} .
$$

In the above equation the integral disappears because $V_{x}$ is arbitrary; moreover, the right-hand side of (A.24) is simplified using the continuity equation, i.e. (A.7), and the referential density, $\hat{\rho}$, disappears because of (A.2a); thus,

$$
\left.\rho \frac{\partial v_{i}}{\partial t}\right|_{x}+\rho w_{j} \frac{\partial v_{i}}{\partial \chi_{j}}=\frac{\partial \sigma_{i i}}{\partial x_{j}}+\rho g_{i} \text { in } R_{x} .
$$

Employing the chain rule and (13) in Section 2.1, and using the symmetry of $\sigma$, it follows

$$
\left.\rho \frac{\partial v_{i}}{\partial t}\right|_{x}+\rho c_{j} \frac{\partial v_{i}}{\partial x_{j}}=\frac{\partial \sigma_{i j}}{\partial x_{j}}+\rho g_{i} \text { in } R_{x} .
$$

\section{Appendix B. Definition of element matrices and vectors}

The matrices necessary to solve the continuity, equilibrium, and mesh updating equations are presented herein. The sum over elements is interpreted as the assembling of the element contributions; $N_{a}, \hat{N}_{a}$, and $N_{a}^{P}$ are the continuous element shape functions for velocity, mesh velocity, and pressure at node ' $a$ ', respectively, and $\bar{k}$ is the the artificial diffusivity. The continuity equation is

$$
\boldsymbol{M}^{P} \dot{\boldsymbol{P}}+\boldsymbol{\eta}^{P}(\boldsymbol{P})+\boldsymbol{G}^{\mathrm{t}} \boldsymbol{v}=\mathbf{0},
$$


where

$$
\begin{aligned}
& M^{P}=\sum_{e} \boldsymbol{M}^{P_{e}}, \quad \boldsymbol{M}^{P_{e}}=\left[M_{I J}^{P}\right], \\
& M_{I J}^{P}=\int_{R_{x}^{e}} \frac{1}{B} N_{a}^{P} N_{b}^{P} \mathrm{~d} R_{x}, \\
& \boldsymbol{\eta}^{P}(\boldsymbol{P})=\sum \boldsymbol{\eta}^{P_{e}}, \quad \boldsymbol{\eta}^{P e}=\left[\eta_{I}^{P}\right], \\
& \eta_{I}^{P}=\int_{R_{x}^{e}} \frac{1}{B} N_{a}^{P} c_{k} \frac{\partial P}{\partial x_{k}} \mathrm{~d} R_{x}, \\
& \boldsymbol{G}=\sum G^{e}, \quad \boldsymbol{G}^{e}=\left[G_{M J}\right] \\
& G_{M J}=\int_{R_{x}^{e}} N_{b}^{P} \frac{\partial N_{c}}{\partial x_{m}} \mathrm{~d} R_{x},
\end{aligned}
$$

and

$$
\begin{array}{ll}
I=(a-1) \mathrm{NEPN} & 1 \leqslant a, b \leqslant \mathrm{NEPN}, \\
J=(b-1) \mathrm{NEPN} \quad \text { with } & 1 \leqslant c \leqslant \mathrm{NEN}, \\
M=(c-1) \mathrm{NEN}+m & 1 \leqslant m \leqslant \mathrm{NSD},
\end{array}
$$

where NEPN, NEN, and NSD are the number of element pressure nodes, the number of velocity nodes per element, and the number of spatial dimensions, respectively. The equilibrium equation is

where

$$
M a+\eta(v)+K_{\mu} v-G P=\mathcal{f}^{e x t},
$$

$$
\begin{aligned}
& M=\sum M^{e}, \quad M^{e}=\left[M_{I J}\right],
\end{aligned}
$$

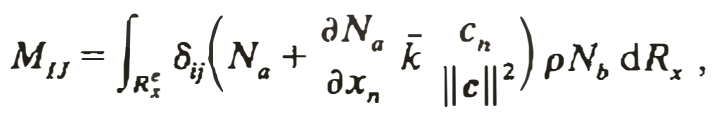

$$
\begin{aligned}
& \boldsymbol{\eta}(\boldsymbol{v})=\sum \boldsymbol{\eta}^{e}, \quad \boldsymbol{\eta}^{e}=\left[\boldsymbol{\eta}_{I}\right], \\
& \eta_{t}=\int_{R_{x}^{e}} \rho\left(N_{a}+\frac{\partial N_{a}}{\partial x_{n}} \bar{k} \frac{c_{n}}{\|c\|^{2}}\right) c_{m} \frac{\partial v_{i}}{\partial x_{m}} \mathrm{~d} R_{x}, \\
& f^{\text {ext }}=\sum f^{\text {exte }}, \quad f^{\text {exte }}=\left[f_{I}^{\text {ext }}\right] \text {, } \\
& f_{I}^{\mathrm{ext}}=\int_{R_{x}^{e}} \rho\left(N_{a}+\begin{array}{cc}
\partial N_{a} & c_{n} \\
\partial x_{n} & \|c\|^{2}
\end{array}\right) g_{i} \mathrm{~d} R_{x},
\end{aligned}
$$

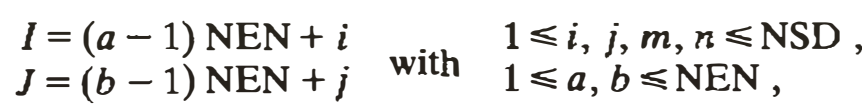

$$
\begin{aligned}
& \boldsymbol{K}_{\mu}=\sum_{e} \boldsymbol{K}_{\mu}^{e}, \quad \boldsymbol{K}_{\mu}^{e}=\int_{R_{x}^{e}} B^{t} D B \mathrm{~d} R_{x}, \\
& \boldsymbol{B}=\left[\boldsymbol{B}_{1} \cdots \boldsymbol{B}_{a} \cdots \boldsymbol{B}_{\mathrm{NEN}}\right],
\end{aligned}
$$


A. Huerta, W.K. Liu, Viscous flow with large free surface motion

$$
\begin{aligned}
\boldsymbol{B}_{a}^{\mathrm{t}} & =\left[\begin{array}{cccccc}
\frac{\partial N_{a}}{\partial x_{1}} & \frac{\partial N_{a}}{\partial x_{2}} & 0 & 0 & 0 & \frac{\partial N_{a}}{\partial x_{3}} \\
0 & \frac{\partial N_{a}}{\partial x_{1}} & \frac{\partial N_{a}}{\partial x_{2}} & 0 & \frac{\partial N_{a}}{\partial x_{3}} & 0 \\
0 & 0 & 0 & \frac{\partial N_{a}}{\partial x_{3}} & \frac{\partial N_{a}}{\partial x_{2}} & \frac{\partial N_{a}}{\partial x_{3}}
\end{array}\right], \\
\boldsymbol{D} & =\left[\begin{array}{rrrrrr}
2 \mu & 0 & 0 & 0 & 0 & 0 \\
0 & \mu & 0 & 0 & 0 & 0 \\
0 & 0 & 2 \mu & 0 & 0 & 0 \\
0 & 0 & 0 & 2 \mu & 0 & 0 \\
0 & 0 & 0 & 0 & \mu & 0 \\
0 & 0 & 0 & 0 & 0 & \mu
\end{array}\right] .
\end{aligned}
$$

The mesh updating equation is

where

$$
\hat{\boldsymbol{M}} \hat{\boldsymbol{v}}+\hat{\boldsymbol{\eta}}(x)-\hat{\boldsymbol{M}} \boldsymbol{v}=\mathbf{0},
$$

$$
\begin{aligned}
& \hat{\boldsymbol{M}}=\sum_{e} \hat{\boldsymbol{M}}^{e}, \quad \hat{\boldsymbol{M}}^{e}=\left[\hat{\boldsymbol{M}}_{I J}\right], \\
& \hat{\boldsymbol{M}}_{I J}=\int_{R_{\chi}^{e}} \delta_{i j}\left(\hat{\boldsymbol{N}}_{a}+\frac{\partial \hat{N}_{a}}{\partial \chi_{n}} \bar{k} \frac{\hat{c}_{n}}{\|\hat{\boldsymbol{c}}\|^{2}}\right) \hat{N}_{b} \mathrm{~d} R_{\chi},
\end{aligned}
$$

and (B.10) hold. The convective term is defined as follows.

(i) Lagrangian-Eulerian matrix method:

$$
\begin{aligned}
& \hat{c}_{i}=\left(\delta_{i j}-\alpha_{i j}\right) v_{j}, \\
& \hat{\boldsymbol{\eta}}(\boldsymbol{x})=\sum_{e} \hat{\boldsymbol{\eta}}^{e}, \quad \hat{\boldsymbol{\eta}}^{e}=\left[\hat{\eta}_{I}\right], \\
& \hat{\boldsymbol{\eta}}_{\boldsymbol{t}}=\int_{R_{x}^{e}}\left(\hat{N}_{a}+\frac{\partial \hat{N}_{a}}{\partial \chi_{n}} k \frac{\hat{c}_{n}}{\|\hat{\boldsymbol{c}}\|^{2}}\right) \hat{c}_{m} \frac{\partial x_{i}}{\partial \chi_{m}} \mathrm{~d} R_{x} .
\end{aligned}
$$

(ii) Mixed formulation: Without any loss of generality, the free surface is assumed perpendicular to the $\chi_{3}$-direction. The cofactors are

$$
\begin{aligned}
& \hat{J}^{33}=\frac{\partial x_{1} \partial x_{2}}{\partial \chi_{1} \partial \chi_{2}}-\frac{\partial x_{1} \partial x_{2}}{\partial \chi_{2} \partial \chi_{1}}, \\
& \hat{J}^{13}=\frac{\partial x_{2}}{\partial \chi_{1}} \frac{\partial x_{3}}{\partial \chi_{2}}-\frac{\partial x_{2}}{\partial \chi_{2}} \frac{\partial x_{3}}{\partial \chi_{1}}, \\
& \hat{J}^{23}=\frac{\partial x_{1} \partial x_{3}}{\partial \chi_{2} \partial \chi_{1}}-\frac{\partial x_{1} \partial x_{3}}{\partial \chi_{1} \partial \chi_{2}} .
\end{aligned}
$$


It should be noted that $x_{3}$ is the only unknown that defines the free surface which is assumed material (i.e. $w_{3}=0$ ). Substituting (B.16) into (37) yields

$$
\begin{aligned}
\left.\frac{\partial x_{3}}{\partial t}\right|_{x} & +\left\{\left(\left(v_{1}-\hat{v}_{1}\right) \frac{\partial x_{2}}{\partial \chi_{2}}-\left(v_{2}-\hat{v}_{2}\right) \frac{\partial x_{1}}{\partial \chi_{2}}\right) / \hat{J}^{33}\right\} \frac{\partial x_{3}}{\partial \chi_{1}} \\
& +\left\{\left(-\left(v_{1}-\hat{v}_{1}\right) \frac{\partial x_{2}}{\partial \chi_{1}}+\left(v_{2}-\hat{v}_{2}\right) \frac{\partial x_{1}}{\partial \chi_{1}}\right) / \hat{J}^{33}\right\} \frac{\partial x_{3}}{\partial \chi_{2}}=0 .
\end{aligned}
$$

Define

$$
\begin{aligned}
& \hat{c}_{1}=\left(\left(v_{1}-\hat{v}_{1}\right) \frac{\partial x_{2}}{\partial \chi_{2}}-\left(v_{2}-\hat{v}_{2}\right) \frac{\partial x_{1}}{\partial \chi_{2}}\right) / \hat{J}^{33}, \\
& \hat{c}_{2}=\left(-\left(v_{1}-\hat{v}_{1}\right) \frac{\partial x_{2}}{\partial \chi_{1}}+\left(v_{2}-\hat{v}_{2}\right) \frac{\partial x_{1}}{\partial \chi_{1}}\right) / \hat{J}^{33}, \\
& \hat{c}_{3}=0,
\end{aligned}
$$

and the convective term in the updating mesh equation can be shown to be

$$
\hat{\eta}_{I}=\int_{R_{x}^{e}}\left(\hat{N}_{a}+\frac{\partial \hat{N}_{a}}{\partial \chi_{n}} \bar{k} \frac{\hat{c}_{n}}{\|\hat{c}\|^{2}}\right) \hat{c}_{m} \frac{\partial x_{i}}{\partial \chi_{m}} \mathrm{~d} R_{x} .
$$

\section{Appendix C. Galerkin formulation for the Lagrange-Euler matrix method}

For the Lagrange-Euler matrix method with a Galerkin formulation the system of partial differential equations goveming the mesh rezoning is

$$
\hat{M} \hat{v}+\hat{\eta}(x)-\hat{M} v=0 .
$$

Similarly to Hughes et al. [22] the integration over the referential domain is done using the Lobatto rule in order to suppress 'diffusion' between Lagrangian and Eulerian nodes. Moreover, it should be noted that the referential domain is fixed in time and therefore the generalized mass matrix is constant and equal to

$$
\begin{aligned}
\hat{M} & =\sum \hat{M}^{e}, \quad \hat{M}^{e}=\left[\hat{M}_{I J}\right], \\
\hat{M}_{I J} & =\int_{R_{x}^{e}} \delta_{i j} \hat{N}_{a} \hat{N}_{b} \mathrm{~d} R_{x} \\
& =J_{a} \omega_{a} \delta_{i j} \delta_{a b},
\end{aligned}
$$

where the same notation is used the as in Appendix $\mathbf{A}$; and $J_{a}$ and $\omega_{a}$ are the Jacobian and weight factor, respectively, associated to the ' $a$ ' node. Moreover, the convective term in (C.1) 
can be transformed to a third-order banded tensor by

with

$$
\hat{\boldsymbol{\eta}}(\boldsymbol{x})=(\hat{L} \boldsymbol{v}) \boldsymbol{x},
$$

$$
\begin{aligned}
\hat{L}=\sum_{e} \hat{L}^{e}, \quad \hat{L}^{e}=\left[\hat{L}_{I J K}\right], & \\
\hat{L}_{I J K} \hat{v}_{K}= & \int_{R_{X}^{e}} \hat{N}_{a} \delta_{i j}\left(\delta_{m n}-\alpha_{m n}\right) v_{n} \frac{\partial \hat{N}_{b}}{\partial \chi_{m}} \mathrm{~d} R_{\chi} \\
& J_{a} \omega_{a} \delta_{i j}\left(\delta_{m n}-\alpha_{m n}(a)\right) \frac{\partial \hat{N}_{b}(a)}{\partial \chi_{m}} v_{n}(a),
\end{aligned}
$$

where

$$
\begin{aligned}
& I=(a-1) \mathrm{NEN}+i \\
& J=(b-1) \mathrm{NEN}+j
\end{aligned} \text { with } \quad \begin{aligned}
& 1 \leqslant i, j, m, n \leqslant \mathrm{NSD} \\
& 1 \leqslant a, b \leqslant \mathrm{NEN} .
\end{aligned}
$$

$K$ varies between one and the number of element variables; and $\boldsymbol{\alpha}(a), \hat{N}_{b}(a)$, and $\boldsymbol{v}(a)$ are the particularizations of the Lagrange-Euler matrix, mesh element shape function, and material velocity, respectively, to the node ' $a$ '. Thus, the third-order banded tensor $\boldsymbol{L}$, constant in time, may be defined by $(\mathrm{C} .4 \mathrm{a})$ and

where

$$
\hat{L}_{I J K} \quad J_{a} \omega_{a} \delta_{i j}\left(\delta_{m n}-\alpha_{m n}(a)\right) \begin{gathered}
\partial \hat{N}_{b}(a) \\
\partial \chi_{m}
\end{gathered}
$$

$$
\begin{array}{ll}
I=(a-1) \mathrm{NEN}+i & 1 \leqslant i, j, m, n \leqslant \mathrm{NSD} \\
J=(b-1) \mathrm{NEN}+j \quad \text { with } & 1 \leqslant a, b \leqslant \mathrm{NEN} . \\
K=(a-1) \mathrm{NEN}+n &
\end{array}
$$

Hence, the mesh updating equation (C.1) may be rewritten as

$$
\hat{M} \hat{v}=\hat{M} v-(\hat{L} v) x
$$

where $\hat{\boldsymbol{L}}$ and $\hat{\boldsymbol{M}}$ can be computed after reading the data, stored and used in every time step.

\section{References}

[1] T. Belytschko and J.M. Kennedy, Computer models for subassembly simulation, Nucl. Engrg. Design 49 (1978) 17-38.

[2] T. Belytschko, J.M. Kennedy and D.F. Schoeberie, Quasi-Eulerian finite element formulation for fluid structure interaction, ASME J. Pressure Vessel Technol. 102 (1980) 62-69.

[3] T. Belytschko and W.K. Liu, Computer methods for transient fluid-structure analysis of nuclear reactors, Nuclear Safety 26 (1985) 14-31.

[4] R.B. Bird, R.C. Annstrong and O. Hassager, Dynamics of Polymeric Liquids, Volume 1: Fluid Mechanics (Wiley, New York, 1977).

[5] G. Brugnot and R. Pochet, Numerical simulation study of avalanches, J. Glaciology 27 (95) (1981) 77-88.

[6] A.N. Brooks and T.J.R. Hughes, Streamline upwind/Petrov-Galerkin formulations for convection dominated 
flows with particular emphasis on the incompressible Navier-Stokes equations, Comput. Meths. Appl. Mech. Engrg. 32 (1982) 199-259.

[7] G.F. Carey and J.T. Oden, Finite Elements: Fluid Mechanics, Volume VI of the Texas Finite Element Series (Prentice-Hall, Englewood Cliffs, NJ, 1986).

[8] C. Chen and J.T. Armbruster, Dam-break wave model: Formulation and verification, ASCE J. Hydraulics Div. 106 (HY5) (1980) 747-767.

[9] J. Donea, Arbitrary Lagrangian-Euletian finite element methods, in: T. Belytschko and T.J.R. Hughes, ed., Computational Methods for Transient Analysis (North-Holland, Amsterdam, 1983) 473-516.

[10] J. Donea, A Taylor-Galerkin method for convective transport problems, Intcrnat. J. Numer. Meths. Engrg. 20 (1984) 101-119.

[11] J. Donea, P. Fasoli-Stella and S. Giuliani, Lagrangian and Eulerian hinite element techniques for transient fluid strncture interaction problems, in: Transactions of the 4th International Conference on Structural Mechanics in Reactor Technology, Paper B1/2, 1977.

[12] R.F. Dressler, Hydraulic resistance effect upon the dam-break functions, J. Res. Nat. Bur. Standards 49 (3) (1952) 217-225.

[13] D.G. Goring, Tsunamis-the propagation of long waves onto a shelf, Dissertation, California Institute of Technology, Pasadena, CA, 1978.

[14] J.C. Heinrich, P.S. Huyakorn, O.C. Zienkiewicz and A.R. Mitchell, An upwind linite clement scheme for two-dimensional convective transport, Internat. J. Numer. Meths. Engrg. 11 (1977) 131-145.

[15] J.C. Heinrich and O.C. Zienkiewicz, The hnite element method and "upwinding" techniques in the numerical solution of convection dominated flow problems, in: T.J.R. Hughes, ed., Finite Elements for Convection Dominated Flow (ASME, New York, 1979) 105-136.

[16] C.W. Hirt, A.A. Amsden and J.L. Cook, An arbitrary Lagrangian Eulerian computing method for all flow speeds, J. Comput. Phys. 14 (1974) 227-253.

[17] A. Huerta, Numerical modeling of slurry mechanics problems. Dissertation, Northwestern University, Evanston, IL, 1987.

[18] A. Huerta and W.K. Liu, Viscous flow structure interaction, J. Pressurc Vessel Technol. 110 (1988) 15-21.

[19] T.J.R. Hughes, A simple scheme for developing upwind finite elements, Internat. J. Numer. Meths. Engrg. 12 (1978) 1359-1365.

[20] T.J.R. Hughes and A.N. Brooks, A theoretical framework for Petrov-Galerkin methods with discontinuous weighting functions: Application to the streamline-upwind procedure, in: R.H. Gallagher, D.H. Norrie, J.T. Oden and O.C. Zienkiewicz, eds., Finite Elements in Fluids (Wiley, New York, 1982) 47-65.

[21] T.J.R. Hughes and W.K. Liu, Implicit-explicit frnite elements in transient analysis, J. Appl. Mech. 45 (1978) 371-378.

[22] T.J.R. Hughes, W.K. Liu and T.K. Zimmerman, Lagrangian-Eulerian finite element formulation for incompressible viscous flows, Comput. Meths. Appl. Mech. Engrg. 29 (1981) 329-349.

[23] T.J.R. Hughes and M. Mallet, A new finite element formulation for computational fluid dynamics: III. The generalized streamline opcrator for multidimensional advective-diffusive systems, Comput. Meths. Appl. Mech. Engrg. 58 (1986) 305-328.

[24] T.J.R. Hughes and T.E. Tezduyar, Finite element methods for first-order hyperbolic systems with particular emphasis on the compressible Euler equations, Comput. Meths. Appl. Mech. Engrg. 45 (1984) 217-284.

[25] K. Hutter and L. Vulliet, Gravity-driven slow creeping flow of a thermoviscous body at elevated temperatures, J. Thermal Stresses (1985) 99-138.

[26] J.K. Jeyapalan, Analysis of flow failures of mine tailings impoundments, Dissertation, University of California, Berkeley, CA, 1980.

[27] M. Keentok, J.F. Milthorpe and E. O'Donovan, On the Shearing zone around rotating vanes in plastic liquids: Theory and experiment, J. Non-Newtonian Fluid Mech. 17 (1985) 23-35.

[28] W.K. Liu, T. Belytschko and H. Chang, An arbitrary Lagrangian-Eulerian finite element method for path-dependent materials, Comput. Meths. Appl. Mech. Engrg. 58 (1986) 227-246.

[29] W.K. Liu and H.G. Chang, Efficient computational procedures for long-time duration fluid-structure interaction problems, ASME J. Pressure Vessel Technol. 106 (1984) 317-322.

[30] W.K. Liu and H.G. Chang, A method of computation for fluid structure interaction, Comput. \& Structures 20 (1-3) (1985) 311-320. 


\section{A. Huerta, W.K. Liu, Viscous flow with large free surface motion}

[31] W.K. Liu, H. Chang and T. Belytschko, Arbitrary Lagrangian-Eulerian Petrov Galerkin finite elements for nonlinear continua, Comput. Methods. Appl. Mech. Engrg. 68 (1988) 259310.

[32] W.K. Liu and J. Gvildys, Fluid structure interactions of tanks with an eccentric core barrel, Comput. Meths. Appl. Mech. Engrg. 58 (1986) 51-57.

[33] W.K. Liu, D. Lain and T. Belytschko, Finite element method for hydrodynamic mass with nonstationary fluid, Comput. Meths. Appl. Mech. Engrg. 44 (1984) 177-211.

[34] W.K. Liu and D. Ma, Computer implementation aspects for fluid structure interaction problems, Comput. Meths. Appl. Mech. Engrg. 31 (1982) 129-148.

[35] R. Löhner, K. Morgan and O.C. Zienkiewicz, The solution of nonlinear hyperbolic equations systems by the finite element method, Internat. J. Numer. Meths. Fluids 4 (1984) 1043-1063.

[36] D.C. Ma, J. Gvildys, Y.W. Chang and W.K. Liu, Seismic behavior of liquid-filled shells, Nucl. Engrg. Design 72 (1982) 437-455.

[37] L.E. Malvern, Introduction to the Mechanics of a Continuous Medium (Prentice-Hall, Englewood Cliffs, NJ, 1965).

[38] K. Muto, Y. Kasai, M. Nakahara and Y. Ishida, Experimental tests on sloshing response of a water pool with submerged blocks, in: S.J. Brown, ed., Proceedings of the 1985 Pressure Vessels and Piping Conference 98-7 (Fluid-Structure Dynamics) (ASME, New York, 1985) 209-214.

[39] W.F. Noh, CEL: A time-depeudent two-space-dimensional coupled Eulerian-Lagrangian code, in: B. Alder, S. Fernbach and M. Rotenberg, eds.; Methods in Computational Physics 3, (Academic Press, New York, 1964).

[40] E.J. O'Donovan and R.I. Tanner, Numerical Study of the Bingham squeeze film problem, J. Non-Newtonian Fluid Mech. 15 (1984) 75-83.

[41] B. Ramaswamy, M. Kawahara and T. Nakayama, Lagrangian finite element method for the analysis of two dimensional sloshing problems, Internat. J. Numer. Meths. Fluids 6 (1986) 659-670.

[42] R.D. Ritchmyer and K.W. Morton, Difference Methods for Initial-Value Problems (Interscience, New York, 2nd. ed., 1967).

[43] A. Ritter, Die Fortpflanzung der Wasserwellew, Z. Vereines Deutscher Ingenieure 36 (33) (1892) 947-954.

[44] J.G. Sakkas and T. Strelkoff, Dimensionless solution of dam-break flood waves, ASCE J. Hydraulics Div. 102 (HY2) (1976) 171-184.

[45] T. Strelkoff, One dimensional equations for open channel flow ASCE J. Hydraulics Div. 95 (HY3) (1969) 861-876.

[46] P. Tangy, M. Fortin and L. Choplin, Finite element simulati n of dip coating, II: Non-Newtonian fluids, Internat. J. Numer. Meths. Fluids. 4 (1984) 459 475.

[47] G.B. Whitham, The effects of hydraulic resistance in the dam-break problem, Proc. Roy. Soc. London Ser. 227 (1955) 399-407.

[48] O.C. Zienkiewicz and P. Bettess, Fluid-structure dynamic interaction and wave forces. An introduction to numerical treatment, Internat. J. Numer. Meths. Engrg. 13 (1978) 1-16. 